\title{
A genericity theorem for algebraic stacks and essential dimension of hypersurfaces
}

Received March 19, 2011 and in revised form December 10, 2011

\begin{abstract}
We compute the essential dimension of the functors Forms $_{n, d}$ and Hypersurf ${ }_{n, d}$ of equivalence classes of homogeneous polynomials in $n$ variables and hypersurfaces in $\mathbb{P}^{n-1}$, respectively, over any base field $k$ of characteristic 0 . Here two polynomials (or hypersurfaces) over a field $K / k$ are considered equivalent if they are related by a linear change of coordinates with coefficients in $K$. Our proof is based on a new Genericity Theorem for algebraic stacks, which is of independent interest. As another application of the Genericity Theorem, we prove a new result on the essential dimension of the stack of (not necessarily smooth) local complete intersection curves.
\end{abstract}

Keywords. Essential dimension, hypersurface, genericity theorem, stack, gerbe

\section{Contents}

1. Introduction . . . . . . . . . . . . . . . . . . . . . . . . . . . . . 1999

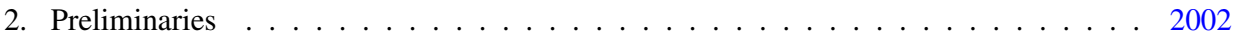

3. Amenable stacks and generic essential dimension . . . . . . . . . . . . . . . . . . 2006

4. Gerbe-like stacks . . . . . . . . . . . . . . . . . . . . . . . 2008

5. The genericity theorem . . . . . . . . . . . . . . . . . . . . . . . . . . 2009

6. Essential dimension of $\mathrm{GL}_{n}$-quotients $\ldots \ldots \ldots \ldots$. . . . . . . . . . . . . 2016

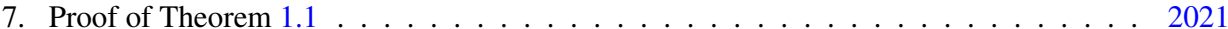

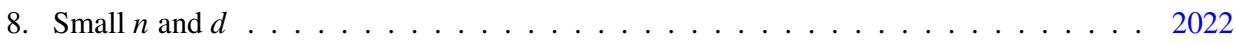

9. Essential dimension of singular curves . . . . . . . . . . . . . . . . . . . . 2024

References . . . . . . . . . . . . . . . . . . . . . . 2025

\section{Introduction}

Let $k$ be a base field of characteristic $0, K / k$ be a field extension, and $F(\underline{x})$ be a homogeneous polynomial (which we call a form) of degree $d$ in the $n$ variables $\underline{x}=\left(x_{1}, \ldots, x_{n}\right)$, with coefficients in $K$. We say that $F$ descends to an intermediate field $k \subset K_{0} \subset K$

Z. Reichstein: Department of Mathematics, University of British Columbia, Vancouver, B.C., Canada V6T 1Z2; e-mail: reichst@math.ubc.ca

A. Vistoli: Scuola Normale Superiore, Piazza dei Cavalieri 7, 56126 Pisa, Italy; e-mail: angelo.vistoli@sns.it

Mathematics Subject Classification (2010): Primary 14A20, 14J70 
if there exists a linear change of coordinates $g \in \mathrm{GL}_{n}(K)$ such that every coefficient of $F(g \cdot \underline{x})$ lies in $K_{0}$.

It is natural to look for a "smallest" subfield $K_{0}$ to which a given form $F(x)$ descends. A minimal such field $K_{0}$ with respect to inclusion may not exist, so we ask instead for the minimal transcendence degree $\operatorname{trdeg}_{k} K_{0}$. This number, called the essential dimension $\operatorname{ed}_{k} F$ of $F$, may be thought of as measuring the "complexity" of $F$. A major goal of this paper is to compute the maximum of $\operatorname{ed}_{k} F$, taken over all fields $K / k$ and all forms $F\left(x_{1}, \ldots, x_{n}\right)$ of degree $d$. This integer, usually called the essential dimension ed $_{k}$ Forms $_{n, d}$ of the functor of forms Forms ${ }_{n, d}$, depends only on $n$ and $d$; it may be viewed as a measure of complexity of all forms of degree $d$ in $n$ variables.

We will also be interested in a variant of this problem, where the form $F(\underline{x}) \in$ $K\left[x_{1}, \ldots, x_{n}\right]$ of degree $d$ is replaced by the hypersurface

$$
H:=\left\{\left(a_{1}: \cdots: a_{n}\right) \mid F\left(a_{1}, \ldots, a_{n}\right)=0\right\}
$$

in $\mathbb{P}^{n-1}$. Here we say that $H$ descends to $K_{0}$ if there exists a linear change of coordinates $g \in \mathrm{GL}_{n}(K)$ and a scalar $c \in K^{*}$ such that every coefficient of $c F(g \cdot \underline{x})$ lies in $K_{0}$. Once again, the essential dimension ed ${ }_{k} H$ of $H$ is defined as the minimal value of $\operatorname{trdeg}_{k} K_{0}$, with the minimum taken over all fields $K_{0} / k$ such that $H$ descends to $K_{0}$. We will be interested in the essential dimension $\operatorname{ed}_{k} \operatorname{Hypersurf}_{n, d}$, defined as the maximal value of $\operatorname{ed}_{k} H$, where the maximum is taken over all $K / k$ and all forms $F(\underline{x}) \in K\left[x_{1}, \ldots, x_{n}\right]$ of degree $d$. Here $H$ is the zero locus of $F$, as in (1.1).

The study of forms played a central role in 19th century algebra. The problems of computing ed Forms $_{n, d}$ and ed Hypersurf $_{n, d}$ are quite natural in this context. However, to the best of our knowledge, these questions did not appear in the literature prior to the (relatively recent) work of G. Berhuy and G. Favi, who showed that ed ${ }_{k}$ Hypersurf $_{3,3}=3$; see [BF04].

In this paper we compute ed Forms $_{n, d}$ and $\operatorname{ed}_{k} \operatorname{Hypersurf}_{n, d}$ for all $n, d \geq 1$. Our main result is as follows.

Theorem 1.1. Assume that $n \geq 2$ and $d \geq 3$ are integers and $(n, d) \neq(2,3),(2,4)$ or $(3,3)$. Then

(a) $\operatorname{ed}_{k}$ Forms $_{n, d}=\left(\begin{array}{c}n+d-1 \\ d\end{array}\right)-n^{2}+\operatorname{cd}\left(\mathrm{GL}_{n} / \mu_{d}\right)+1$.

(b) ed $_{k}$ Hypersurf $_{n, d}=\left(\begin{array}{c}n+d-1 \\ d\end{array}\right)-n^{2}+\operatorname{cd}\left(\mathrm{GL}_{n} / \mu_{d}\right)$.

The values of ed Forms $_{n, d}$ and ed Hypersurf $_{n, d}$ for $n, d \geq 1$ not covered by Theorem 1.1 are computed in Section 8; the results are summarized in the following table.

\begin{tabular}{cccc}
\hline$n$ & $d$ & ed $_{k}$ Forms $_{n, d}$ & ed $_{k}$ Hypersurf $_{n, d}$ \\
\hline arbitrary & 1 & 0 & 0 \\
1 & $\geq 2$ & 1 & 0 \\
arbitrary & 2 & $n$ & $n-1$ \\
2 & 3 & 2 & 1 \\
2 & 4 & 3 & 2 \\
3 & 3 & 4 & 3 \\
\hline
\end{tabular}


The quantity $\operatorname{cd}\left(\mathrm{GL}_{n} / \mu_{d}\right)$ which appears in the statement of Theorem 1.1 is the canonical dimension of the algebraic group $\mathrm{GL}_{n} / \mu_{d}$. For the definition and basic properties of canonical dimension we refer the reader to Section 2.2; see also [BR05, KaMe06] for a more extensive treatment of this notion. The exact value of $\operatorname{cd}\left(\mathrm{GL}_{n} / \mu_{d}\right)$ is known in the case where $e:=\operatorname{gcd}(n, d)$ is a prime power $p^{j}$. In this case

$$
\operatorname{cd}\left(\mathrm{GL}_{n} / \mu_{d}\right)= \begin{cases}p^{i}-1 & \text { if } j>0 \\ 0 & \text { otherwise }\end{cases}
$$

where $p^{i}$ is the highest power of $p$ dividing $n$; see [BR05, Section 11]. More generally, suppose $e=p_{1}^{j_{1}} \ldots p_{r}^{j_{r}}$ is the prime decomposition of $e$ (with $j_{1}, \ldots, j_{r} \geq 1$ ) and $p_{s}^{i_{s}}$ is the highest power of $p_{s}$ dividing $n$. A conjecture of J.-L. Colliot-Thélène, N. A. Karpenko, and A. S. Merkurjev [CTKM07, (2)] implies that

$$
\operatorname{cd}\left(\mathrm{GL}_{n} / \mu_{d}\right)=\sum_{s=1}^{r}\left(p_{s}^{i_{s}}-1\right)
$$

This has only been proved if $e$ is a prime power (as above) or $n=6$ [CTKM07, Theorem 1.3]. In these two cases Theorem 1.1 gives the exact value of ed $_{k}$ Forms $_{n, d}$ and $\operatorname{ed}_{k}$ Hypersurf $_{n, d}$. For other $n$ and $d$ Theorem 1.1 reduces the problems of computing $\operatorname{ed}_{k}$ Forms $_{n, d}$ and ed Hypersurf $_{n, d}$ to the problem of computing the canonical dimension $\operatorname{cd}\left(\mathrm{GL}_{n} / \mu_{d}\right)$. For partial results on the latter problem, see [BR05, Section 11].

The notions of essential dimension for forms and hypersurfaces are particular cases of Merkurjev's general definition of essential dimension of a functor [BF03]. A special case of this, upon which our approach is based, is the essential dimension of an algebraic stack. For background material on this notion we refer the reader to [BRV11]. In particular, $\operatorname{ed}_{k}$ Forms $_{n, d}=\operatorname{ed}_{k}\left[A_{n, d} / \mathrm{GL}_{n}\right]$ and $\operatorname{ed}_{k} \operatorname{Hypersurf}_{n, d}=\operatorname{ed}_{k}\left[\mathbb{P}\left(A_{n, d}\right) / \mathrm{GL}_{n}\right]$, where $A_{n, d}$ is the $\left(\begin{array}{c}n+d-1 \\ d\end{array}\right)$-dimensional affine space of forms of degree $d$ in $n$ variables and $\mathbb{P}\left(A_{n, d}\right)$ is the associated $\left(\begin{array}{c}n+d-1 \\ d\end{array}\right)-1$-dimensional projective space of degree $d$ hypersurfaces in $\mathbb{P}^{n-1}$. (Here, as in the rest of the paper, we will follow the classical convention of defining the projectivization $\mathbb{P}(V)$ of a vector space $V$ over $k$ as the projective space of lines in $V$, that is, as $\operatorname{Proj}_{\operatorname{Sym}_{k}} V^{\vee}$. In the present context, this seems more natural than Grothendieck's convention of defining $\mathbb{P}(V)$ as $\operatorname{Proj} \operatorname{Sym}_{k} V$.) The group GL $\mathrm{G}_{n}$ naturally acts on these spaces, and $\left[A_{n, d} / \mathrm{GL}_{n}\right]$ and $\left[\mathbb{P}\left(A_{n, d}\right) / \mathrm{GL}_{n}\right]$ denote the quotient stacks for these actions; see [BRV11, Example 2.6].

The essential dimension of the "generic hypersurface" of degree $d$ in $\mathbb{P}^{n-1}$, i.e., of the hypersurface $H_{\text {gen }}$ cut out by the "generic form"

$$
F_{\text {gen }}\left(x_{1}, \ldots, x_{n}\right)=\sum_{i_{1}+\cdots+i_{n}=d} a_{i_{1}, \ldots, i_{n}} x_{1}^{i_{1}} \ldots x_{n}^{i_{n}}=0,
$$

where $a_{i_{1}, \ldots, i_{n}}$ are independent variables and $K$ is the field generated by these variables over $k$, was computed in [BR05, Sections 14-15]. The question of computing the essential dimension of the generic form $F_{\text {gen }}$ itself was left open in [BR05]. For $n$ and $d$ as in Theorem 1.1 we will show that $\mathrm{ed}_{k} F_{\text {gen }}=\operatorname{ed}_{k} H_{\text {gen }}+1$; see Proposition 3.4. 
The key new ingredient in the proof of Theorem 1.1 is the following "Genericity Theorem". Let $\mathcal{X}$ be a connected algebraic stack with quasi-affine diagonal that is smooth of finite type over $k$, in which the automorphism groups are generically finite (for the sake of brevity, we say that $\mathcal{X}$ is amenable). Then we can define the generic essential dimension of $\mathcal{X}$, denoted by $\operatorname{ged}_{k} \mathcal{X}$, as the supremum of the essential dimensions of the dominant points Spec $K \rightarrow \mathcal{X}$. If $\mathcal{X}$ is Deligne-Mumford, that is, if all stabilizers are finite, then $\operatorname{ed}_{k} \mathcal{X}=\operatorname{ged}_{k} \mathcal{X}$; see [BRV11, Theorem 6.1]. This result, which we called the Genericity Theorem for Deligne-Mumford stacks in [BRV11], is not sufficient for the applications in the present paper. Here we prove the following stronger theorem conjectured in [BRV11, Question 6.6].

Theorem 1.2. Let $\mathcal{X}$ be an amenable stack over $k$. Let $L$ be a field extension of $k$, and $\xi$ be an object of $\mathcal{X}(\operatorname{Spec} L)$, such that the automorphism group scheme $\underline{\mathrm{Aut}}_{L} \xi$ is reductive. Then

$$
\operatorname{ed}_{k} \xi \leq \operatorname{ged}_{k} \mathcal{X}
$$

In particular, if the automorphism group of any object of $\mathcal{X}$ defined over a field is reductive, then $\operatorname{ed}_{k} \mathcal{X}=\operatorname{ged}_{k} \mathcal{X}$

Note that Theorem 1.2 fails if the stabilizers are not required to be reductive (see [BRV11, Example 6.5(b)]), even though a weaker statement may be true in this setting (see Conjecture 5.4). We also remark that the locus of points with reductive stabilizer is constructible but not necessarily open in $\mathcal{X}$. Thus for the purpose of proving Theorem 1.2 it does not suffice to consider the case where all stabilizers are reductive.

Theorem 1.2 implies, in particular, that if the automorphism group of a form $f\left(x_{1}, \ldots, x_{n}\right)$ is reductive then $\operatorname{ed}_{k} f \leq \operatorname{ed}_{k} F_{\text {gen }}$. To complete the proof of Theorem 1.1(a) we supplement this inequality with additional computations, carried out in Section 6 , which show that forms $f\left(x_{1}, \ldots, x_{n}\right)$ whose automorphism group is not reductive have low essential dimension; for a precise statement, see Theorem 6.3. The proof of Theorem 1.1(b) is more delicate because the quotient stack $\left[\mathbb{P}\left(A_{n, d}\right) / \mathrm{GL}_{n}\right]$ is not amenable, so the Genericity Theorem cannot be applied to it directly. We get around this difficulty in Section 7 by relating $\operatorname{ed}_{k}\left[\mathbb{P}\left(A_{n, d}\right) / \mathrm{GL}_{n}\right]$ to the essential dimension of the amenable stack $\left[\mathbb{P}\left(A_{n, d}\right) / \mathrm{PGL}_{n}\right]$.

In the last section we use our Genericity Theorem 1.2 to prove a new result on the essential dimension of the stack of (not necessarily smooth) local complete intersection curves, strengthening [BRV11, Theorem 7.3].

\section{Preliminaries}

\subsection{Special groups}

A linear algebraic group scheme $G$ over $k$ is said to be special if for every extension $K / k$ we have $\mathrm{H}^{1}(K, G)=\{1\}$. Special groups were studied by Serre [CGS58, Exposé 1] and classified by Grothendieck [CGS58, Exposé 5] (over an algebraically closed field of characteristic 0 ). Note that $G$ is special if and only if $\operatorname{ed}_{k} G=0$; see [TV13, Proposition 4.3]. 
The group $\mathrm{GL}_{n}$ is special by Hilbert's Theorem 90, and so is $\mathrm{SL}_{n}$. Direct products of special groups are easily seen to be special. Moreover, in characteristic 0 the group $G$ is special if and only if the Levi subgroup of $G$ (which is isomorphic to $G / \mathrm{R}_{\mathrm{u}} G$ ) is special; see [San81, Theorem 1.13]. Here $\mathrm{R}_{\mathrm{u}} G$ denotes the unipotent radical of $G$. We record the following fact for future reference.

Let $A$ be a non-zero nilpotent $n \times n$-matrix with entries in $k$ and $G_{A}$ be the image of the map $\mathbb{G}_{\mathrm{a}} \rightarrow \mathrm{GL}_{n}$ given by $t \mapsto \exp (t A)$. Note that this map is algebraic, since only finitely many terms in the power series expansion of $\exp (t A)$ are non-zero.

\section{Lemma 2.1.}

(a) The centralizer $C$ of $A$ (or equivalently, of $G_{A}$ ) in $\mathrm{GL}_{n}$ is special.

(b) The normalizer $N$ of $G_{A}$ in $\mathrm{GL}_{n}$ is special.

Proof. (a) By [Jan04, Propositions 3.10 and 3.8.1], $C$ is a semidirect product $U \rtimes H$, where $U \triangleleft C$ is unipotent and $H$ is the direct product of general linear groups $\mathrm{GL}_{r}$ for various $r \geq 0$; cf. also [McN06, Section 2]. Thus $H=$ Levi subgroup of $C$ is special, and part (a) follows.

(b) The normalizer $N$ acts on $G_{A} \simeq \mathbb{G}_{\text {a }}$ by conjugation. This gives rise to a homomorphism $\pi: N \rightarrow \mathbb{G}_{\mathrm{m}}=\underline{\text { Aut }}_{k} \mathbb{G}_{\mathrm{a}}$ whose kernel is the centralizer $C=C_{\mathrm{GL}_{n}}(A)$. We claim that $\pi$ is surjective, i.e., the sequence

$$
1 \rightarrow C \rightarrow N \stackrel{\pi}{\rightarrow} \mathbb{G}_{\mathrm{m}} \rightarrow 1
$$

is exact. If this claim is established, the long non-abelian cohomology sequence associated with this short exact sequence shows that $\mathrm{H}^{1}(K, N)=\{1\}$ for every field $K / k$, i.e., $N$ is special, as desired.

Now observe that our claim is equivalent to the assertion that for every $t \in \bar{k}$ there exists a $B \in \mathrm{M}_{n}(\bar{k})$ such that $B^{-1} A B=t A$. To prove this assertion, we may assume that $A$ is a single Jordan block

$$
A=\left(\begin{array}{ccccc}
0 & 1 & 0 & \ldots & 0 \\
0 & 0 & 1 & \ldots & 0 \\
\vdots & \vdots & \ddots & \vdots & \\
0 & 0 & \ldots & 0 & 1 \\
0 & 0 & \ldots & 0 & 0
\end{array}\right)
$$

In this case we can take $B$ to be the diagonal matrix $B=\operatorname{diag}\left(1, t, t^{2}, \ldots\right)$. This completes the proof of the claim and the lemma.

\subsection{Canonical dimension}

Let $K$ be a field and $X$ be either a geometrically integral smooth complete $K$-scheme of finite type or a $G$-torsor for some connected linear algebraic $K$-group $G$. The canonical dimension $\operatorname{cd} X$ of $X$ is the minimal value of $\operatorname{dim} Y$, where $Y$ ranges over all integral closed $K$-subschemes of $X$ admitting a rational map $X \rightarrow Y$ defined over $K$. Equivalent 
definitions via generic splitting fields and determination functions can be found in [BR05, $\mathrm{KaMe}$ 06].

If we fix a base field $k$ and an algebraic $k$-group $G$, the maximal value of $\operatorname{cd} X$ as $K$ ranges over all field extensions $K / k$ and $X \rightarrow$ Spec $K$ ranges over all $G_{K}$-torsors, is denoted by $\operatorname{cd} G$. Moreover, $\operatorname{cd} G=\operatorname{cd} X_{\mathrm{ver}}$, where $X_{\mathrm{ver}} \rightarrow \operatorname{Spec} K_{\mathrm{ver}}$ is a versal $G$ torsor. In particular, we can construct a versal $G$-torsor by starting with a generically free linear representation $V$ of $G$ defined over $k$ and setting $K_{\mathrm{ver}}:=k(V)^{G}$. Then $V$ has a $G$-invariant open subset $U$ which is the total space of a $G$-torsor $U \rightarrow B$, where $k(B)=k(V)^{G}$. Restricting to the generic point $\eta$ of $B$, we obtain a versal torsor $X_{\mathrm{ver}}:=$

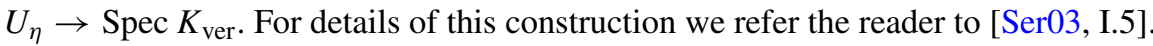

\section{Lemma 2.2.}

(a) Let $X_{1}$ and $X_{2}$ be Brauer-equivalent Brauer-Severi varieties over a field $K / k$. Then

$$
\operatorname{cd} X_{1}=\operatorname{cd} X_{2} \text {. }
$$

In other words, the canonical dimension $\mathrm{cd} \alpha$ of a Brauer class $\alpha \in \mathrm{H}^{2}\left(K, \mathbb{G}_{\mathrm{m}}\right)$ is well defined.

(b) Let $G=\mathrm{GL}_{n}$ or $\mathrm{SL}_{n}$ and let $C$ be a central subgroup scheme of $G$. Then for any field $K / k$ and any $(G / C)$-torsor $X \rightarrow \operatorname{Spec} K$ we have $\operatorname{cd} X=\operatorname{cd} \alpha$, where $\alpha$ $i$ is the image of the class of $X$ under the coboundary map $\partial_{K}: \mathrm{H}^{1}(K, G / C) \rightarrow$ $\mathrm{H}^{2}(L, C) \subseteq \mathrm{H}^{2}\left(K, \mathbb{G}_{\mathrm{m}}\right)$ induced by the exact sequence $1 \rightarrow C \rightarrow G \rightarrow G / C \rightarrow 1$.

(c) Let $K / k$ be a field extension and $\alpha \in \mathrm{H}^{2}\left(K, \mathbb{G}_{\mathrm{m}}\right)$ be a Brauer class of index dividing $n$ and exponent dividing $d$. Then $\operatorname{cd} \alpha \leq \operatorname{cd}\left(\mathrm{GL}_{n} / \mu_{d}\right)$.

Proof. (a) follows from the fact that $X_{1}$ and $X_{2}$ have the same splitting fields $L / K$; see [BR05, Section 10] or [KaMe06, Section 2].

(b) By Hilbert's Theorem 90, $G$ is special, i.e., $\mathrm{H}^{1}(L, G)=\{1\}$ for any field $L$. Hence, the coboundary map $\partial_{L}: \mathrm{H}^{1}(L, G / C) \rightarrow \mathrm{H}^{2}(L, C)$ has trivial kernel for any $L$. In other words, $X$ and $\alpha$ have the same splitting fields, and part (b) follows (cf. [BR05, Lemma 10.2]).

(c) By our assumption, $\alpha$ lies in the image of the coboundary map

$$
\partial_{K}: \mathrm{H}^{1}\left(K, \mathrm{GL}_{n} / \mu_{d}\right) \rightarrow \mathrm{H}^{2}(K, C)
$$

cf., e.g., [BR05, Lemma 2.6]. Part (c) now follows from part (b).

The following result will be used repeatedly.

\section{Proposition 2.3.}

(a) Let $\mathcal{X} \rightarrow \operatorname{Spec} K$ be a $\mathbb{G}_{\mathrm{m}}$-gerbe over a field $K$. Denote the class of this gerbe in $\mathrm{H}^{2}\left(K, \mathbb{G}_{\mathrm{m}}\right)$ by $\alpha$. Then $\operatorname{ed}_{K} \mathcal{X}=\operatorname{cd} \alpha$.

(b) Let $e \geq 2$ be an integer and $\mathcal{X} \rightarrow$ Spec $K$ be a $\mu_{e}$-gerbe over a field $K$. Denote the class of this gerbe in $\mathrm{H}^{2}\left(K, \mu_{e}\right)$ by $\beta$. Then $\operatorname{ed}_{K} \mathcal{X}=\operatorname{cd} \beta+1$.

Proof. See [BRV11, Theorem 4.1]. 


\subsection{Gerbes and Brauer classes}

Let $\phi: \mathcal{X} \rightarrow \overline{\mathcal{X}}$ be a $\mathbb{G}_{\mathrm{m}}$-gerbe over a stack $\overline{\mathcal{X}}$. If $L$ is a field and $\xi \in \overline{\mathcal{X}}(L)$ then, pulling back $\mathcal{X}$ to Spec $L$ we obtain a $\mathbb{G}_{\mathrm{m}}$-gerbe $\mathcal{X}_{\xi}$ over $L$. We will denote by $\operatorname{ind}\left(\mathcal{X}_{\xi}\right)$ and $\exp \left(\mathcal{X}_{\xi}\right)$ the index and exponent of the Brauer class of $\mathcal{X}_{\xi}$. The following lower semicontinuity properties of ind and $\exp$ (as functions of $\xi$ ) will be used in the proof of Theorem 1.1(b).

Lemma 2.4. Let $\phi: \mathcal{X} \rightarrow \overline{\mathcal{X}}$ be a $\mathbb{G}_{\mathrm{m}}$-gerbe over an integral regular algebraic stack $\overline{\mathcal{X}}$, as above. Assume further that $\overline{\mathcal{X}}$ is generically a scheme, with generic point $\eta:$ Spec $K \rightarrow \overline{\mathcal{X}}$. Then for any field $L / k$ and any $\xi \in \overline{\mathcal{X}}(L)$,

(a) $\operatorname{ind}\left(\mathcal{X}_{\xi}\right)$ divides $\operatorname{ind}\left(\mathcal{X}_{\eta}\right)$, and

(b) $\exp \left(\mathcal{X}_{\xi}\right)$ divides $\exp \left(\mathcal{X}_{\eta}\right)$.

Proof. (a) The key fact we will use is that if $B$ is a Brauer-Severi variety over a field $L$ then ind $(B)$ divides $d$ if and only if $B$ has a linear subspace of dimension $d-1$ defined over $L$; see [Art82, Proposition 3.4].

By [LMB00a, Theorem 6.3] there exists a smooth map $T \rightarrow \overline{\mathcal{X}}$ such that $\xi$ lifts to a point $\operatorname{Spec} L \rightarrow T$. We may assume that $T$ is affine, regular and integral. The index of the pullback of $\mathcal{X}_{\eta}$ to the function field $k(T)$ divides ind $\left(\mathcal{X}_{\eta}\right)$; hence we can replace $\overline{\mathcal{X}}$ with $T$, and assume that $\overline{\mathcal{X}}=T$ is an affine regular integral variety. The étale cohomology group $\mathrm{H}^{2}\left(T, \mathbb{G}_{\mathrm{m}}\right)$ is torsion, because $T$ is regular; hence, by a well known result of $\mathrm{O}$. Gabber [Gab81] the class of $\mathcal{X}$ is represented by a Brauer-Severi scheme $P \rightarrow T$.

Let $d$ be the index ind $\left(\overline{\mathcal{X}}_{\eta}\right):=\operatorname{ind}\left(P_{\eta}\right)$ and $\operatorname{Gr}(P, d-1) \rightarrow T$ be the Grassmannian bundle of linear subspaces of dimension $d-1$ in $P$. The generic fiber $\operatorname{Gr}(P, n-1)_{\eta}$ has a $K$-rational point; this gives rise to a section $U \rightarrow \operatorname{Gr}(P, n-1)$ over some open subscheme $U$ of $T$. Let $Y$ be the complement of $U$ in $T$. If our point $\xi: \operatorname{Spec} L \rightarrow \overline{\mathcal{X}}$ lands in $U$, then the pullback $P_{\xi}$ has a linear subspace of dimension $d-1$ defined over $L$, and we are done. Thus we may assume that $\xi \in Y(L)$. The morphism $\xi$ : Spec $L \rightarrow T$ extends to a morphism Spec $R \rightarrow T$, where $R$ is a DVR with residue field $L$, such that the generic point of $\operatorname{Spec} R$ lands in $U$. The pullback $\operatorname{Gr}(P, d-1)_{R}$ of $\operatorname{Gr}(P, d-1)$ to Spec $R$ then has a section over the generic point. By the valuative criterion of properness this section extends to a section $\operatorname{Spec} R \rightarrow \operatorname{Gr}(P, d-1)$. Specializing to the closed point of $\operatorname{Spec} R$, we obtain a desired section $\operatorname{Spec} L \rightarrow \operatorname{Gr}(P, d-1)$. This shows that $P_{\xi}$ has degree dividing $d$, as claimed.

(b) Set $e:=\exp \left(\mathcal{X}_{\xi}\right)$ and apply part (a) to the $e^{\text {th }}$ power $\mathcal{Y}$ of the gerbe $\mathcal{X}$. Since $\mathcal{Y}_{\eta}$ is trivial (i.e., has index 1), so is $\mathcal{Y}_{\xi}$. But $\mathcal{Y}_{\xi}$ is the $e^{\text {th }}$ power of the class of $\mathcal{X}_{\xi}$, and we are done.

An alternative proof of part (b) is based on the fact that a Brauer-Severi variety $B \rightarrow$ Spec $L$ over a field $L$ has index dividing $e$ if and only if $P$ contains a hypersurface of degree $e$ defined over $L$; see [Art82, (5.2)]. We may thus proceed exactly as in the proof of part (a), with the same $T$ and $P \rightarrow T$, but using the Hilbert scheme $H(P, e) \rightarrow T$ of hypersurfaces of degree $e$ in $P$ instead of the Grassmannian. 
Remark 2.5. It was pointed out to us by the referee that after reducing to the case where $\overline{\mathcal{X}}=T$ is an affine regular integral variety, an alternative proof can be given by using the theory of twisted sheaves.

\section{Amenable stacks and generic essential dimension}

Definition 3.1. Let $\mathcal{X}$ be an algebraic stack over $k$. We say that $\mathcal{X}$ is amenable if the following conditions hold:

(a) $\mathcal{X}$ is integral with quasi-affine diagonal.

(b) $\mathcal{X}$ is smooth (and in particular, is locally of finite type) over $k$.

(c) There exists a non-empty open substack of $\mathcal{X}$ that is a Deligne-Mumford stack.

Any irreducible algebraic stack has a generic gerbe, the residual gerbe at any dominant point Spec $K \rightarrow \mathcal{X}$ [LMB00a, §11]. For amenable stacks, there is an alternative description. Let $\mathcal{X}$ be an amenable stack over $k$, and $\mathcal{U}$ a non-empty open substack which is Deligne-Mumford. After shrinking $\mathcal{U}$, we may assume that the inertia stack $\mathcal{I}_{\mathcal{U}}$ is finite over $\mathcal{U}$. Let $\mathbf{U}$ be the moduli space of $\mathcal{U}$, whose existence is proved in [KeMo97], and let $k(\mathbf{X})$ be its residue field. The generic gerbe $\mathcal{X}_{k(\mathbf{X})} \rightarrow \operatorname{Spec} k(\mathbf{X})$ is then the fiber product $\operatorname{Spec} k(\mathbf{X}) \times_{\mathbf{U}} \mathcal{U}$. The $\operatorname{dimension} \operatorname{dim} \mathcal{X}$ is the dimension of $\mathcal{U}$, or, equivalently, the dimension of $\mathbf{U}$.

Example 3.2. Consider the action of a linear algebraic group defined over $k$ on a smooth integral $k$-scheme $X$, locally of finite type. Then the quotient stack $[X / G]$ is amenable if and only if the stabilizer $\operatorname{Stab}_{G}(x)$ of a general point $x \in X$ is finite.

Of particular interest to us will be the $\mathrm{GL}_{n}$-actions on $A_{n, d}$, the $\left(\begin{array}{c}n+d-1 \\ d\end{array}\right)$-dimensional affine space of forms of degree $d$ in $n$ variables, and $\mathbb{P}\left(A_{n, d}\right)$, the $\left(\begin{array}{c}n+d-1 \\ d\end{array}\right)-1$ dimensional projective space of degree $d$ hypersurfaces in $\mathbb{P}^{n-1}$, as well as the $\mathrm{PGL}_{n^{-}}$ action on $\mathbb{P}\left(A_{n, d}\right)$.

Since the center of $\mathrm{GL}_{n}$ acts trivially on $\mathbb{P}\left(A_{n, d}\right)$, the stack $\left[\mathbb{P}\left(A_{n, d}\right) / \mathrm{GL}_{n}\right]$ is not amenable. On the other hand, it is classically known that the stabilizer $\operatorname{Stab}_{\mathrm{PGL}_{n}}(h)$ of any smooth hypersurface $h \subset \mathbb{P}^{n-1}$ of degree $d \geq 3$ is finite; see, e.g., [OS78, Theorem 2.1] or $[\mathrm{MM} 64]$. From this we deduce that the stacks $\left[\mathbb{P}\left(A_{n, d}\right) / \mathrm{PGL}_{n}\right]$ and $\left[A_{n, d} / \mathrm{GL}_{n}\right]$ are both amenable for any $n \geq 2$ and $d \geq 3$.

Moreover, if $n \geq 2, d \geq 3$ and $(n, d) \neq(2,3),(2,4)$ or $(3,3)$ then the stabilizer of a general hypersurface in $\mathbb{P}^{n-1}$ of degree $d$ is trivial; see [MM64]. For these values of $n$ and $d$ the quotient stack $\left[\mathbb{P}\left(A_{n, d}\right) / \mathrm{PGL}_{n}\right]$ is generically a scheme of dimension

$$
\operatorname{dim} \mathbb{P}\left(A_{n, d}\right)-\operatorname{dim} \mathrm{PGL}_{n}=\left(\begin{array}{c}
n+d-1 \\
d
\end{array}\right)-n^{2} .
$$

Definition 3.3. The generic essential dimension of an amenable stack $\mathcal{X}$ is

$$
\operatorname{ged}_{k} \mathcal{X}:=\operatorname{ed}_{k(\mathbf{X})} \mathcal{X}_{k(\mathbf{X})}+\operatorname{dim} \mathcal{X}
$$


Alternatively, $\operatorname{ged}_{k} \mathcal{X}$ is the supremum of the essential dimension of $\zeta \in \mathcal{X}(K)$, taken over all field extensions $K / k$ and all dominant $\zeta$ : Spec $K \rightarrow \mathcal{X}$. By the Genericity Theorem for Deligne-Mumford stacks [BRV11, Theorem 6.1], we see that $\operatorname{ged}_{k} \mathcal{X}$ is the essential dimension of any open substack of $\mathcal{X}$ that is a Deligne-Mumford stack.

We will now compute the generic essential dimension of the quotient stacks $\left[A_{n, d} / \mathrm{GL}_{n}\right]$ and $\left[\mathbb{P}\left(A_{n, d}\right) / \mathrm{GL}_{n}\right]$ for $n$ and $d$ as in the statement of Theorem 1.1. Recall that $\operatorname{ged}_{k}\left[A_{n, d} / \mathrm{GL}_{n}\right]=\operatorname{ed}_{k} F_{\text {gen }}$ and $\operatorname{ged}_{k}\left[\mathbb{P}\left(A_{n, d}\right) / \mathrm{GL}_{n}\right]=\operatorname{ed}_{k} H_{\text {gen }}$, where $F_{\text {gen }}$ is the generic form of degree $d$ in $n$ variables and $H_{\text {gen }}$ is the generic hypersurface, as in (1.3).

Proposition 3.4. Let $n \geq 2$ and $d \geq 3$ be integers. Assume further that $(n, d) \neq(2,3)$, $(2,4)$ or $(3,3)$. Then

(a) $\operatorname{ged}_{k}\left[\mathbb{P}\left(A_{n, d}\right) / \mathrm{GL}_{n}\right]=\left(\begin{array}{c}n+d-1 \\ d\end{array}\right)-n^{2}+\operatorname{cd}\left(\mathrm{GL}_{n} / \mu_{d}\right)$.

(b) $\operatorname{ged}_{k}\left[A_{n, d} / \mathrm{GL}_{n}\right]=\left(\begin{array}{c}n+d-1 \\ d\end{array}\right)-n^{2}+\operatorname{cd}\left(\mathrm{GL}_{n} / \mu_{d}\right)+1$.

Part (a) was previously known; see [BR05, Theorem 15.1]. Part (b) answers an open question from [BR05, Remark 14.8].

Proof. Let $\mathcal{X}=\left[\mathbb{P}\left(A_{n, d}\right) / \mathrm{GL}_{n}\right], \mathcal{Y}=\left[A_{n, d} \backslash\{0\} / \mathrm{GL}_{n}\right]$, and $\overline{\mathcal{X}}=\left[\mathbb{P}\left(A_{n, d}\right) / \mathrm{PGL}_{n}\right]$. Consider the diagram

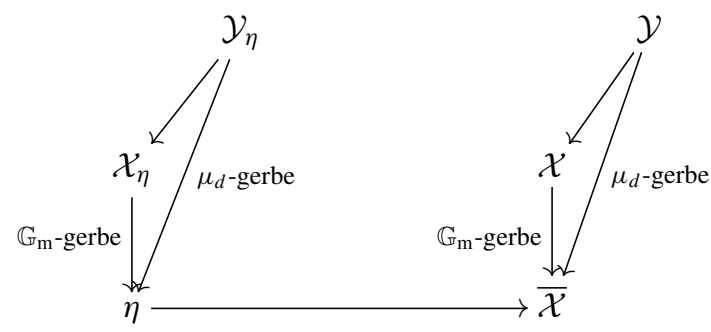

For $n$ and $d$ as in the statement of the proposition, $\left[\mathbb{P}\left(A_{n, d}\right) / \mathrm{PGL}_{n}\right]$ is generically a scheme (see Example 3.2). Denote the generic point of this scheme by $\eta$ and its function field by $k(\eta)$. The pullbacks $\mathcal{Y}_{k(\eta)}$ and $\mathcal{X}_{k(\eta)}$ are, respectively, a $\mu_{d}$-gerbe and a $\mathbb{G}_{\mathrm{m}}$-gerbe over $k(\eta)$; these two gerbes give rise to the same class $\alpha \in \mathrm{H}^{2}\left(k(\eta), \mu_{d}\right) \subset$ $\mathrm{H}^{2}\left(k(\eta), \mathbb{G}_{\mathrm{m}}\right)$. By Proposition 2.3,

$$
\operatorname{ed}_{k(\eta)} \mathcal{X}_{k(\eta)}=\operatorname{cd} \alpha \quad \text { and } \quad \operatorname{ed}_{k(\eta)} \mathcal{Y}_{k(\eta)}=\operatorname{cd} \alpha+1
$$

Since

it remains to show that

$$
\operatorname{trdeg}_{k} k(\eta)=\left(\begin{array}{c}
n+d-1 \\
d
\end{array}\right)-n^{2},
$$

$$
\operatorname{cd} \alpha=\operatorname{cd}\left(\mathrm{GL}_{n} / \mu_{d}\right) \text {. }
$$

The action of $G=\mathrm{GL}_{n} / \mu_{d}$ on $A_{n, d}$ is linear and generically free. Thus it gives rise to a versal $G$-torsor $t \in \mathrm{H}^{1}(k(\eta), G)$, and $\alpha$ is the image of $t$ under the natural coboundary map $\mathrm{H}^{1}(k(\eta), G) \rightarrow \mathrm{H}^{2}\left(k(\eta), \mu_{d}\right)$ associated with the exact sequence $1 \rightarrow \mu_{d} \rightarrow$ $\mathrm{GL}_{n} \rightarrow G \rightarrow 1$. As explained in Section 2.2, $\mathrm{cd} t=\mathrm{cd}\left(\mathrm{GL}_{n} / \mu_{d}\right)$. On the other hand, by Lemma 2.2(b), $\operatorname{cd} \alpha=\operatorname{cd} t$, and (3.1) follows. 


\section{Gerbe-like stacks}

The purpose of the next two sections is to prove the Genericity Theorem 1.2. The proof of the genericity theorem for Deligne-Mumford stacks in [BRV11] relied on a stronger form of genericity for gerbes; see [BRV11, Theorem 5.13]. Our proof of Theorem 1.2 will follow a similar pattern, except that instead of working with gerbes we will need to work in the more general setting of gerbe-like stacks, defined below. The main result of this section, Theorem 4.6, is a strong form of genericity for gerbe-like stacks.

Definition 4.1. A Deligne-Mumford stack $\mathcal{X}$ is gerbe-like if its inertia stack $\mathcal{I}_{\mathcal{X}}$ is étale over $\mathcal{X}$.

If $\mathcal{X}$ is an algebraic stack, the gerbe-like part $\mathcal{X}^{0}$ of $\mathcal{X}$ is the largest open substack of $\mathcal{X}$ that is Deligne-Mumford and gerbe-like.

Remark 4.2. If an algebraic stack $\mathcal{X}$ is Deligne-Mumford, then the inertia stack $\mathcal{I}_{\mathcal{X}} \rightarrow \mathcal{X}$ is unramified. Hence, if $\mathcal{X}$ is also reduced then by generic flatness the gerbe-like part $\mathcal{X}^{0}$ of $\mathcal{X}$ is dense in $\mathcal{X}$.

Lemma 4.3. Let $\mathcal{X}$ be a reduced Deligne-Mumford stack. Suppose that the inertia stack $\mathcal{I}_{\mathcal{X}}$ is finite and étale over $\mathcal{X}$. Then $\mathcal{X}$ is a proper étale gerbe over an algebraic space.

Remark 4.4. The condition that $\mathcal{X}$ be reduced can be eliminated. However, it makes the proof marginally simpler, and will be satisfied in all cases of interest to us in this paper.

Proof. Let $\mathbf{X}$ be the moduli space of $\mathcal{X}$; we claim that $\mathcal{X}$ is a proper étale gerbe over $\mathbf{X}$. This is a local problem in the étale topology of $\mathbf{X}$. Hence, after passing to an étale covering of $\mathbf{X}$, we may assume that $\mathbf{X}$ is a connected scheme, and there exists a finite reduced connected scheme $U$, with a finite group $G$ acting on $U$, such that $\mathcal{X}=[U / G]$. The pullback of $\mathcal{I}_{\mathcal{X}}$ to $U$ is the closed subscheme of $G \times U$ defined as representing the functor of pairs $(g, u)$ with $g u=u$. The fact that this pullback is étale over $U$ translates into the condition that the order of the stabilizer of a geometric point is locally constant on $U$ (and hence, constant, since $U$ is connected). That is, that every geometric point of $U$ lies in the same number of closed subschemes $U^{g}=\{u \mid g u=u\}$, where $g \in G(\bar{k})$. This is only possible if for every $g \in G(\bar{k}), U^{g}=\emptyset$ or $U^{g}=U$. In other words, there exists a subgroup $H$ of $G$ that acts trivially on $U$ (this subgroup is necessarily normal and defined over $k$ ), and the induced action of $G / H$ on $U$ is free. Then $U /(G / H)=\mathbf{X}$; hence $U$ is étale over $\mathbf{X}$, and $\mathcal{X}=[U / G]$ is a gerbe banded by $H$ over $\mathbf{X}$.

Lemma 4.5. Suppose that $\mathcal{X}$ is a gerbe-like Deligne-Mumford stack, and $\mathcal{Y} \rightarrow \mathcal{X}$ a representable unramified morphism. Then $\mathcal{Y}$ is also gerbe-like.

Proof. The inertia stack $\mathcal{I}_{\mathcal{X}}$ of a stack $\mathcal{X}$ is the fiber product $\mathcal{X} \times \mathcal{X} \times \mathcal{X}$. We have a diagram 


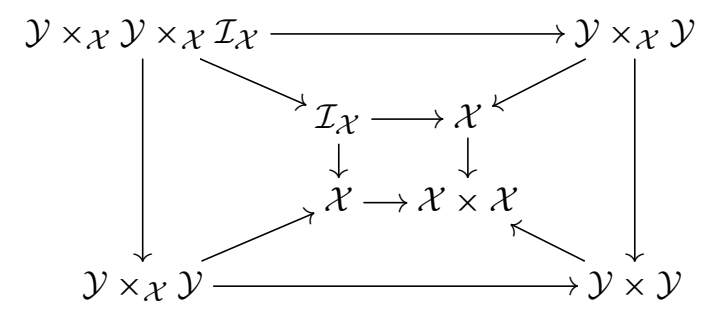

in which all the squares are cartesian. This implies the equality

$$
\mathcal{Y} \times \mathcal{X} \mathcal{Y} \times \mathcal{X} \mathcal{I}_{\mathcal{X}}=(\mathcal{Y} \times \mathcal{X} \mathcal{Y}) \times \mathcal{X}(\mathcal{Y} \times \mathcal{X} \mathcal{Y})
$$

which in turn tells us that $(\mathcal{Y} \times \mathcal{X} \mathcal{Y}) \times \mathcal{X}(\mathcal{Y} \times \mathcal{X} \mathcal{Y})$ is étale over $\mathcal{Y} \times \mathcal{X} \mathcal{Y}$. The hypotheses on $\mathcal{Y} \rightarrow \mathcal{X}$ imply that the diagonal $\mathcal{Y} \rightarrow \mathcal{Y} \times \mathcal{X} \mathcal{Y}$ is an open embedding. Thus $\mathcal{I}_{\mathcal{Y}}=\mathcal{Y}_{\mathcal{Y} \times \mathcal{Y}}$ is an open substack of $(\mathcal{Y} \times \mathcal{X} \mathcal{Y}) \times \mathcal{X}(\mathcal{Y} \times \mathcal{X} \mathcal{Y})$, so it is étale over $\mathcal{Y} \times \mathcal{X} \mathcal{Y}$, hence it is étale over $\mathcal{Y}$, as claimed.

From this and the results in [BRV11], it is easy to deduce the following. Given a field $L / k$ and $\xi \in \mathcal{X}(\operatorname{Spec} L)$, we denote by $\operatorname{codim} \mathcal{X} \xi$ the codimension of the closure of the image of the corresponding morphism $\operatorname{Spec} L \rightarrow \mathcal{X}$.

Theorem 4.6. Let $\mathcal{X}$ be an integral gerbe-like Deligne-Mumford stack which is smooth of finite type over a field $k$. Let $L$ be an extension of $k$ and $\xi \in \mathcal{X}(\operatorname{Spec} L)$. Then

$$
\operatorname{ed} \mathcal{X} \xi \leq \operatorname{ed}_{k(\mathbf{X})} \mathcal{X}_{k(\mathbf{X})}+\operatorname{dim} \mathbf{X}-\operatorname{codim}_{\mathcal{X}} \xi
$$

Proof. If the inertia stack $\mathcal{I}_{\mathcal{X}}$ is finite over $\mathcal{X}$, then, by Lemma $4.3, \mathcal{X}$ is an étale proper gerbe over a smooth $k$-scheme, and the statement reduces to [BRV11, Theorem 5.13]. In the general case, from [BRV11, Lemma 6.4] we deduce the existence of an étale representable morphism $\mathcal{Y} \rightarrow \mathcal{X}$ such that $\mathcal{Y}$ is an integral Deligne-Mumford stack with finite inertia, and the morphism Spec $L \rightarrow \mathcal{Y}$ factors through $\mathcal{Y}$. By Lemmas 4.3 and 4.5, the stack $\mathcal{Y}$ is a proper étale gerbe over a smooth algebraic space, hence [BRV11, Theorem 5.13] can be applied to it. Let $\eta \in \operatorname{Spec} \mathcal{Y}$ be a point in $\mathcal{Y}(L)$ mapping to $\xi$. Then

$$
\operatorname{ed} \mathcal{X} \eta \leq \operatorname{ed}_{k(\mathbf{Y})} \mathcal{Y}_{k(\mathbf{Y})}+\operatorname{dim} \mathbf{Y}-\operatorname{codim}_{\mathcal{Y}} \eta
$$

and

$$
\operatorname{ed}_{k} \eta \geq \operatorname{ed}_{k} \xi, \quad \operatorname{ed}_{k(\mathcal{X})} \mathcal{X}_{k(\mathbf{X})} \geq \operatorname{ed}_{k(\mathbf{Y})} \mathcal{Y}_{k(\mathbf{Y})} \quad \text { and } \quad \operatorname{codim}_{\mathcal{X}} \xi=\operatorname{codim}_{\mathcal{Y}} \eta
$$

and the inequality of Theorem 4.6 follows from (4.1).

\section{The genericity theorem}

We now proceed with the proof of Theorem 1.2. We will assume throughout that $L$ is a field extension of $k$ and $\xi:$ Spec $L \rightarrow \mathcal{X}$ is an object of $\mathcal{X}(L)$. We will denote by $\mathcal{Y}$ the closure of the image of the morphism $\xi$, with its reduced stack structure. The stack $\mathcal{Y}$ is integral, and since char $k=0, \mathcal{Y}$ is generically smooth. Let $\pi: \mathcal{M} \rightarrow \mathbb{A}_{k}^{1}$ be deformation to the normal bundle of $\mathcal{Y}$ inside $\mathcal{X}$. Recall that $\pi^{-1}\left(\mathbb{A}_{k}^{1} \backslash\{0\}\right)=\mathcal{X} \times \operatorname{Spec} k\left(\mathbb{A}_{k}^{1} \backslash\{0\}\right)$, while $\pi^{-1}(0)$ is isomorphic to the normal bundle $\mathcal{N}$ of $\mathcal{Y}$ in $\mathcal{X}$. 
Denote by $\mathcal{M}^{0}$ the substack of $\mathcal{M}$ whose geometric points have stabilizers of minimal order. Note that $\mathcal{M}^{0}$ is well-defined and open in $\mathcal{M}$ because $X$ is amenable (and so is $\mathcal{M}$ ).

Lemma 5.1. $\mathcal{M}^{0} \cap \mathcal{N} \neq \varnothing$.

Theorem 1.2 follows from Lemma 5.1 and Theorem 4.6 by the same argument as in [BRV11, Theorem 6.1]. This argument is quite short, and we reproduce it here for the sake of completeness.

Set $\mathcal{N}^{0}:=\mathcal{M}^{0} \cap \mathcal{N}$. Then the fiber product $\operatorname{Spec} L \times \mathcal{Y} \mathcal{N}$ is a vector bundle over $\operatorname{Spec} L$, and $\operatorname{Spec} L \times \mathcal{X} \mathcal{N}^{0}$ is a non-empty open subscheme. Hence $\xi: \operatorname{Spec} L \rightarrow \mathcal{Y}$ can be lifted to $\mathcal{N}^{0}$; this gives an object $\eta$ of $\mathcal{N}^{0}$ (Spec $\left.L\right)$ mapping to $\xi$ in $\mathcal{Y}$. Clearly the essential dimension of $\xi$ as an object of $\mathcal{X}$ is the same as its essential dimension as an object of $\mathcal{Y}$, and $\operatorname{ed}_{k} \xi \leq \operatorname{ed}_{k} \eta$. Let us apply Theorem 4.6 to the gerbe $\mathcal{M}^{0}$. The function field of the moduli space $\mathbf{M}$ of $\mathcal{M}$ is $k(\mathbf{X})(t)$, and its generic gerbe is $\mathcal{X}_{k(\mathbf{X})(t)}$; by [BRV11, Proposition 2.8], we have $\operatorname{ed}_{k(\mathbf{X})(t)} \mathcal{X}_{k(\mathbf{X})(t)} \leq \operatorname{ed}_{k(\mathbf{X})} \mathcal{X}_{k(\mathbf{X})}$. The composite Spec $L \rightarrow \mathcal{N}^{0} \subseteq \mathcal{M}^{0}$ has codimension at least 1 , hence we obtain

$$
\operatorname{ed}_{k} \xi<\operatorname{ed}_{k(\mathbf{X})(t)} \mathcal{X}_{k(\mathbf{X})(t)}+\operatorname{dim} \mathcal{M}=\operatorname{ed}_{k(\mathbf{X})} \mathcal{X}_{k(\mathbf{X})}+\operatorname{dim} \mathcal{X}+1
$$

This concludes the proof.

The rest of this section will be devoted to the proof of Lemma 5.1. Our proof is quite complicated, so we will split it into several steps.

We will begin with some easy preliminary reductions. For the purpose of proving Lemma 5.1, we may base-change to the algebraic closure of $k$, i.e., assume that $k$ is algebraically closed. By deleting the singular locus of $\mathcal{Y}$, we may assume that $\mathcal{Y}$ is smooth. Moreover, after passing to dense open substacks, we may assume that the inertia stack $\mathcal{I}_{\mathcal{Y}}$ is flat over $\mathcal{Y}$, and that all geometric fibers are reductive, and have the same numbers of connected components.

\subsection{The linear case}

Let us suppose that $X$ is a finite-dimensional representation of a linearly reductive algebraic group $G$ and $Y \subseteq X$ is a subrepresentation. (Here we identify $X$ with the affine space $\operatorname{Spec}\left(\operatorname{Sym}_{k}^{\bullet} X^{\vee}\right)$ and similarly for $Y$.) Since $G$ is reductive, we have a $G$-equivariant splitting $X \simeq Y \oplus Y^{\prime}$. Set $\mathcal{X}:=[X / G]$ and $\mathcal{Y}:=[Y / G]$. Assume that the generic stabilizer of the action of $G$ on $X$ is finite. Then $\mathcal{X}$ is amenable, and $\mathcal{Y} \subseteq \mathcal{X}$ is a closed integral substack.

It is easy to see that the deformation to the normal bundle $M$ of $Y$ in $X$ is $G$ equivariantly isomorphic to $X \times{ }_{k} \mathbb{A}_{k}^{1}$ (where the group $G$ acts trivially on $\mathbb{A}_{k}^{1}$ ); the projection

$$
Y \times Y^{\prime} \times_{k} \mathbb{A}_{k}^{1}=X \times_{k} \mathbb{A}_{k}^{1} \simeq M \rightarrow X \times_{k} \mathbb{A}_{k}^{1}
$$

is given by the formula $\left(y, y^{\prime}, t\right) \mapsto\left(t y, y^{\prime}, t\right)$. The deformation to the normal bundle $\mathcal{M}$ of $\mathcal{Y}$ is $[M / G]=[X / G] \times \times_{k} \mathbb{A}_{k}^{1}$; hence $\mathcal{M}^{0}=\mathcal{X}^{0} \times_{k} \mathbb{A}_{k}^{1}$, and it is obvious that $\mathcal{M}^{0} \cap \mathcal{N} \neq \emptyset$.

The proof in the general case will be reduced to this by a formal slice argument. 


\subsection{Formal linearization}

Let $y_{0}:$ Spec $k \rightarrow \mathcal{Y}$ be a general rational point. The next step is to show that near $y_{0}$ the stack $\mathcal{X}$ has formally the structure of a quotient of a finite-dimensional representation.

The residual gerbe $\mathcal{G}_{y_{0}} \rightarrow$ Spec $k$ [LMB00a, §11] admits a section Spec $k \rightarrow \mathcal{G}_{y_{0}}$, since $k$ is algebraically closed. Thus if $G:=\underline{\text { Aut }}_{k} y_{0}$ is the automorphism group scheme of $y_{0}$, we have $\mathcal{G}_{y_{0}} \simeq \mathcal{B}_{k} G$. The embedding of stacks $\mathcal{B}_{k} G \rightarrow \mathcal{Y}$ is of finite type. By Zariski's Main Theorem for stacks [LMB00a, Théorème 16.5] it is a locally closed embedding.

Let $U \rightarrow \mathcal{X}$ be a smooth morphism, where $U$ is a scheme, together with a lifting $u_{0}:$ Spec $k \rightarrow U$ of $y_{0}$. Denote by $\mathcal{X}_{n}$ the $n^{\text {th }}$ infinitesimal neighborhood of $\mathcal{B}_{k} G$ inside $\mathcal{X}$ : in other words, if $\mathcal{U}$ is an open substack of $\mathcal{X}$ containing $\mathcal{B}_{k} G$ as a closed substack, and we denote by $I$ the sheaf of ideals of $\mathcal{B}_{k} G$ inside $\mathcal{U}$, then $\mathcal{X}_{n}$ is the closed substack of $\mathcal{X}$ defined by the sheaf of ideals $I^{n+1}$. In particular, $\mathcal{X}_{0}=\mathcal{B}_{k} G$.

Lemma 5.2. There exists a finite-dimensional representation $X$ of $G$ with finite generic stabilizers and a trivial subrepresentation $Y \subseteq X$, with the following property. If we denote by $X_{n}$ and $Y_{n}$ the $n^{\text {th }}$ infinitesimal neighborhoods of the origin, there is a sequence of isomorphisms $\mathcal{X}_{n} \simeq\left[X_{n} / G\right]$, compatible with the embeddings $\mathcal{X}_{n} \subseteq \mathcal{X}_{n+1}$ and $\left[X_{n} / G\right] \subseteq\left[X_{n+1} / G\right]$, that induce isomorphisms of $\mathcal{Y}_{n}$ with $\left[Y_{n} / G\right]$

Furthermore, denote by $\widehat{X}$ the spectrum of the completion of the local ring of $X$ at the origin. Then there exists a smooth morphism $U \rightarrow \mathcal{X}$ with a closed point $u_{0} \in U$ mapping to $y_{0}$ in $\mathcal{Y}$, and an isomorphism of $\widehat{X}$ with the spectrum $\widehat{U}$ of the completion of the local ring of $U$ at $u_{0}$, such that

(a) the sequence of composites $X_{n} \rightarrow\left[X_{n} / G\right] \simeq \mathcal{X}_{n} \subseteq \mathcal{X}$ is obtained by restriction from the composite morphism $\widehat{X} \simeq \widehat{U} \rightarrow U \rightarrow \mathcal{X}$, and

(b) the inverse image of $Y$ in $\widehat{X}$ corresponds to the inverse image of $\mathcal{Y}$ in $\widehat{U}$.

Proof. By [Alp09, Propositions 5.1 and 5.2] the tautological $G$-torsor $P_{0}:=\operatorname{Spec} k \rightarrow$ $\mathcal{B}_{k} G$ extends to a $G$-torsor $P_{n} \rightarrow \mathcal{X}_{n}$ in such a way that the restriction of $P_{n+1}$ to $\mathcal{X}_{n} \subseteq \mathcal{X}_{n+1}$ is isomorphic to $P_{n}$. Each of the stacks $P_{n}$ is a scheme, because its reduced substack is; in fact, $P_{n}$ must be the spectrum of a local artinian $k$-algebra $R_{n}$. Clearly, $\mathcal{X}_{n}=\left[P_{n} / G\right]$.

If we denote by $V$ the maximal ideal of $R_{1}$, then $R_{1}=k \oplus V$; the action of $G$ on $R_{1}$ induces a linear action of $G$ on $V$. The space $V$ is isomorphic to $I / I^{2}$, which is a coherent sheaf on $\mathcal{B}_{k} G$, i.e., a representation of $G$. In turn, $I / I^{2}$ is the cotangent space of deformations of Spec $k \rightarrow \mathcal{X}$, that is, the dual to the space of isomorphism classes of liftings Spec $k[\epsilon] \rightarrow \mathcal{X}$ of Spec $k \rightarrow \mathcal{X}$. Here, as usual, $k[\epsilon]$ denotes the ring of dual numbers $k[x] /\left(x^{2}\right)$.

The homomorphism $R_{n+1} \rightarrow R_{n}$ induced by the embedding $P_{n} \simeq P_{n+1} \mid \mathcal{X}_{n} \subseteq P_{n+1}$ is surjective; its kernel is the ideal $I^{n} R_{n+1}$. Denote by $R$ the projective limit $\lim _{n} R_{n}$. (Note that, $G$ acts, by definition, on each of $R_{n}$. In general, this does not induce an algebraic group action of $G$ on $R$, unless $G$ is finite. If we had a $G$-action on $R$, this would make the proof conceptually much simpler.) If $x_{1}, \ldots, x_{n}$ is a set of elements of $R$ that project to a basis for $V$ in $R_{1}$, the ring $R$ is a quotient of the power series ring 
$k\left[\left[x_{1}, \ldots, x_{n}\right]\right.$ by an ideal $J$ contained in $\mathfrak{m}_{R}^{2}$. We claim that $J=0$, i.e., $R$ is a power series ring. For this, it is enough to check that $R$ is formally smooth over $k$, or, in other words, that if $A$ is a local artinian $k$-algebra with residue field $k$ and $B$ is a quotient of $A$, any homomorphism of $k$-algebras $R \rightarrow B$ lifts to a homomorphism $R \rightarrow A$. Take $n \gg 0$; then $R \rightarrow B$ factors through $R_{n}$. Consider the composite

$$
\operatorname{Spec} B \rightarrow \operatorname{Spec} R_{n} \rightarrow \mathcal{X}_{n} \subseteq \mathcal{X}
$$

since $\mathcal{X}$ is smooth, deformations are unobstructed, i.e., this morphism extends to $\operatorname{Spec} A$ $\rightarrow \mathcal{X}$. If $n \gg 0$, this factors as $\operatorname{Spec} A \rightarrow \mathcal{X}_{n} \subseteq \mathcal{X}$; and since Spec $R_{n}$ is smooth over $\mathcal{X}_{n}$, as it is a $G$-torsor, the section $\operatorname{Spec} B \rightarrow \operatorname{Spec} R_{n}$ of $\operatorname{Spec} B \rightarrow \mathcal{X}_{n}$ lifts to a section Spec $A \rightarrow$ Spec $R_{n}$, giving the desired extension $R \rightarrow R_{n} \rightarrow A$.

Suppose that $U \rightarrow \mathcal{X}$ a smooth morphism, where $U$ is a scheme, with a lifting $u_{0}:$ Spec $k \rightarrow U$ of $y_{0}$. Let us assume that $U$ is minimal at $u_{0}$, or, in other words, that the tangent space of $U$ at $u_{0}$ maps isomorphically onto the deformation space of $\mathcal{X}$ at $y_{0}$. Since $U$ is smooth over $\mathcal{X}$, the morphisms Spec $R_{n} \rightarrow \mathcal{X}$ lift to a compatible system of morphisms Spec $R_{n} \rightarrow U$, sending Spec $k$ into $u_{0}$; these yield a morphism $\operatorname{Spec} R \rightarrow U$, inducing a homomorphism of $k$-algebras $\widehat{\mathcal{O}}_{U, u_{0}} \rightarrow$ Spec $R$. This is a homomorphism of power series algebras over $k$ which induces an isomorphism of tangent spaces. By Nakayama's lemma, it is an isomorphism. This shows that the morphisms $\operatorname{Spec} R_{n} \rightarrow \mathcal{X}$ patch to a flat morphism $\operatorname{Spec} R \rightarrow \mathcal{X}$.

Let $d$ be the codimension of $\mathcal{Y}$ in $\mathcal{X}$ at the point $y_{0}$. After a base change in $R=k \llbracket x_{1}, \ldots, x_{n} \rrbracket$, we may assume that the inverse image of the ideal of $\mathcal{Y}$ in $\mathcal{X}$ is $\left(x_{1}, \ldots, x_{d}\right)$. Denote by $X$ the scheme corresponding to the dual of the vector space $V=\left\langle x_{1}, \ldots, x_{n}\right\rangle$. That is, $X:=\operatorname{Spec}_{\operatorname{Sym}_{k}}^{\bullet}$. Let $Y$ the linear subscheme defined by the ideal $\left(x_{1}, \ldots, x_{d}\right)$. Then $\widehat{X}=\operatorname{Spec} R$; the representation $X$ has all the required properties, except that we have not yet proved that the action of $G$ on $X$ has finite generic stabilizers, and the representation $Y$ is trivial.

To do this, let us denote by $I$ the pullback of the inertia stack of $[X / G]$ to $X$. In other words, $I$ is the subscheme of $G \times \operatorname{Spec} k X$ defined by the equation $g x=x$. We need to show that $I$ is generically finite over $X$. Since $I$ is a group scheme over $X$, it has equidimensional fibers, hence it is enough to show that there is an étale neighborhood $I^{\prime} \rightarrow I$ of the pair $\left(1, u_{0}\right)$ in $I$ which is generically finite over $X$. The inverse image of $\mathcal{X}_{n}$ in $U$ is the $n^{\text {th }}$ infinitesimal neighborhood $U_{n}$ of $u_{0}$ in $U$. Denote by $J$ the pullback of the inertia stack of $\mathcal{X}$ to $U$; we have isomorphisms $X_{n} \simeq U_{n}$, and compatible isomorphisms of the pullbacks of $I$ and $J$ to $X_{n}$ and $U_{n}$ respectively. These induce an isomorphism of the completions of $I$ and $J$ at $\left(1, x_{0}\right)$ and $\left(1, u_{0}\right)$ respectively; by Artin approximation, the morphisms $I \rightarrow X$ and $J \rightarrow U$ are étale-locally equivalent at $\left(1, x_{0}\right)$ and $\left(1, u_{0}\right)$. Since $J$ is generically finite over $U$ it follows that the action of $G$ on $X$ has generically finite stabilizers. Also, this implies that the stabilizer of a general closed point of $Y$ is isomorphic to the isomorphism group scheme of a general point of $\operatorname{Spec} k \rightarrow \mathcal{Y}$. This stabilizer has the same dimension and the same number of connected components as $G$. Hence it equals $G$, i.e., the action of $G$ on $Y$ is trivial, as claimed. 


\subsection{The formal structure of the deformation to the normal bundle}

Here we use the result on the formal structure of $\mathcal{X}$ around $y_{0}$ to conclude that $\mathcal{M}$ has the desired formal structure around the fiber of $\mathcal{M}$ over $y_{0}$. The difficulty is that deformation to the normal cone does not commute with non-flat pullbacks, and $\mathcal{M}_{n}$ (defined below) is not obtained directly from $\mathcal{Y}_{n} \subseteq \mathcal{X}_{n}$. We will get around this point with a formal scheme argument, which relies on the following fact.

Suppose that $X$ is a noetherian scheme, and $Y \subseteq X$ a closed subscheme. Let $\tilde{X}$ be the completion of $X$ along $Y$. By construction, there is a morphism of locally ringed spaces $\widetilde{X} \rightarrow X$. It is easy to check that it is flat, by reducing to the affine case $X=\operatorname{Spec} A$, and using the standard fact that the completion $A$ along the ideal of $Y$ is flat over $A$.

Denote by $\mathcal{M}_{n}$ the inverse image of $\mathcal{X}_{n} \times \operatorname{Spec} k \mathbb{A}_{k}^{1}$ in $\mathcal{M}$, and by $M_{n}$ the inverse image of $X_{n} \times \operatorname{Speck} \mathbb{A}_{k}^{1}$ in $M$.

Lemma 5.3. There is a sequence of isomorphisms $\mathcal{M}_{n} \simeq\left[M_{n} / G\right]$ compatible with the isomorphisms $\mathcal{X}_{n} \simeq\left[X_{n} / G\right]$, and with the identity on $\mathbb{A}_{k}^{1}$.

Proof. Set

$$
\begin{aligned}
R & :=U \times \mathcal{X} U, \quad S:=X \times[X / G] X=G \times \times_{\operatorname{Spec} k} X, \\
R_{n} & :=U_{n} \times \mathcal{X}_{n} U_{n}, \quad S_{n}:=X_{n} \times_{\left[X_{n} / G\right]} X_{n}=G \times \operatorname{Spec} k X_{n} .
\end{aligned}
$$

The compatible isomorphisms $\phi_{n}: X_{n} \simeq U_{n}$ and $\mathcal{X}_{n} \simeq\left[X_{n} / G\right]$ yield isomorphisms of schemes in groupoids of $R_{n} \rightrightarrows U_{n}$ with $S_{n} \rightrightarrows X_{n}$, for each $n \geq 0$.

Denote by $I_{U}$ the sheaf of ideals of the inverse image of $\mathcal{Y} \times\{0\} \subseteq \mathcal{X} \times \mathbb{A}^{1}$ in $U \times \mathbb{A}^{1}$, and by $I_{R}$ the sheaf of ideals of its inverse image in $R \times \mathbb{A}^{1}$. Also denote by $J_{U}$ the sheaf of ideals of $\mathcal{Y} \times\{0\} \subseteq \mathcal{X} \times\{0\}$ in $U \times\{0\}$, pushed forward to $U \times \mathbb{A}^{1}$, and by $J_{R}$ the sheaf of ideals of its inverse image in $R \times\{0\}$, pushed forward to $R \times \mathbb{A}^{1}$. There are natural surjections $I_{U} \rightarrow J_{U}$ and $I_{R} \rightarrow J_{R}$. Set

$$
\begin{aligned}
U^{\prime}:=\operatorname{Proj}_{U \times \mathbb{A}^{1}}\left(\bigoplus_{m=0}^{\infty} I_{U}^{m}\right), \quad R^{\prime}:=\operatorname{Proj}_{R \times \mathbb{A}^{1}}\left(\bigoplus_{m=0}^{\infty} I_{R}^{m}\right), \\
U^{\prime \prime}:=\operatorname{Proj}_{U \times \mathbb{A}^{1}}\left(\bigoplus_{m=0}^{\infty} J_{U}^{m}\right), \quad R^{\prime \prime}:=\operatorname{Proj}_{R \times \mathbb{A}^{1}}\left(\bigoplus_{m=0}^{\infty} J_{R}^{m}\right) .
\end{aligned}
$$

Then $R^{\prime} \rightrightarrows U^{\prime}$ is a scheme in groupoids, $R^{\prime \prime} \rightrightarrows U^{\prime \prime}$ is a closed subgroupoid, and the difference groupoid $R^{\prime} \backslash R^{\prime \prime} \rightrightarrows U^{\prime} \backslash U^{\prime \prime}$ gives a smooth presentation of $\mathcal{M}$. Let us denote by $U_{n}^{\prime}$ and $U_{n}^{\prime \prime}$ the inverse images of $U_{n}$ in $U^{\prime}$ and $U^{\prime \prime}$, and by $R_{n}^{\prime}$ and $R_{n}^{\prime \prime}$ the inverse images of $U_{n} \times U_{n}$ in $R^{\prime}$ and $R^{\prime \prime}$. Then the groupoid $R_{n}^{\prime} \backslash R_{n}^{\prime \prime} \rightrightarrows U_{n}^{\prime} \backslash U_{n}^{\prime \prime}$ gives a smooth presentation of $\mathcal{M}_{n}$; furthermore, we have

$$
\begin{aligned}
& U_{n}^{\prime}=\operatorname{Proj}_{U_{n} \times \mathbb{A}^{1}}\left(\bigoplus_{m=0}^{\infty} I_{U}^{m} \otimes \mathcal{O}_{U \times \mathbb{A}^{1}} \mathcal{O}_{U_{n} \times \mathbb{A}^{1}}\right), \\
& R_{n}^{\prime}=\operatorname{Proj}_{R_{n} \times \mathbb{A}^{1}}\left(\bigoplus_{m=0}^{\infty} I_{R}^{m} \otimes_{\mathcal{O}_{U \times \mathbb{A}^{1}}} \mathcal{O}_{R_{n} \times \mathbb{A}^{1}}\right),
\end{aligned}
$$




$$
\begin{aligned}
& U_{n}^{\prime \prime}=\operatorname{Proj}_{U_{n} \times \mathbb{A}^{1}}\left(\bigoplus_{m=0}^{\infty} J_{U}^{m} \otimes_{\mathcal{O}_{R \times \mathbb{A}^{1}}} \mathcal{O}_{U_{n} \times \mathbb{A}^{1}}\right), \\
& R_{n}^{\prime \prime}=\operatorname{Proj}_{R_{n} \times \mathbb{A}^{1}}\left(\bigoplus_{m=0}^{\infty} J_{R}^{m} \otimes_{\mathcal{O}_{R \times \mathbb{A}^{1}}} \mathcal{O}_{R_{n} \times \mathbb{A}^{1}}\right) .
\end{aligned}
$$

In a completely analogous manner, denote by $I_{X}$ the sheaf of ideals of $Y \times\{0\}$ in $U \times \mathbb{A}^{1}$, and by $I_{S}$ the sheaf of ideals of its inverse image in $S \times \mathbb{A}^{1}$. Also denote by $J_{X}$ the sheaf of ideals of $Y \times\{0\}$ in $X \times\{0\}$, pushed forward to $X \times \mathbb{A}^{1}$, and by $J_{S}$ the sheaf of ideals of its inverse image in $S \times\{0\}$, pushed forward to $S \times \mathbb{A}^{1}$. Set

$$
\begin{aligned}
X_{n}^{\prime} & :=\operatorname{Proj}_{X_{n} \times \mathbb{A}^{1}}\left(\bigoplus_{m=0}^{\infty} I_{X}^{m} \otimes_{\mathcal{O}_{X \times \mathbb{A}^{1}}} \mathcal{O}_{X_{n} \times \mathbb{A}^{1}}\right), \\
S_{n}^{\prime} & :=\operatorname{Proj}_{S_{n} \times \mathbb{A}^{1}}\left(\bigoplus_{m=0}^{\infty} I_{S}^{m} \otimes_{\mathcal{O}_{S \times \mathbb{A}^{1}}} \mathcal{O}_{S_{n} \times \mathbb{A}^{1}}\right), \\
X_{n}^{\prime \prime} & :=\operatorname{Proj}_{U \times \mathbb{A}^{1}}\left(\bigoplus_{m=0}^{\infty} J_{U}^{m} \otimes_{\mathcal{O}_{R \times \mathbb{A}^{1}}} \mathcal{O}_{R_{n} \times \mathbb{A}^{1}}\right), \\
S_{n}^{\prime \prime} & :=\operatorname{Proj}_{R \times \mathbb{A}^{1}}\left(\bigoplus_{m=0}^{\infty} J_{R}^{m} \otimes_{\mathcal{O}_{R \times \mathbb{A}^{1}}} \mathcal{O}_{R_{n} \times \mathbb{A}^{1}}\right)
\end{aligned}
$$

By the same argument as before, we see that $\left[M_{n} / G\right]$ has a smooth presentation $S_{n}^{\prime} \backslash S_{n}^{\prime \prime} \rightrightarrows$ $X_{n}^{\prime} \backslash X_{n}^{\prime \prime}$; hence, to complete the proof we need to establish the existence of isomorphisms $U_{n}^{\prime} \simeq X_{n}^{\prime}$ and $R_{n}^{\prime} \simeq S_{n}^{\prime}$, compatible with the groupoid structures, the isomorphisms $\phi_{n}: U_{n} \simeq X_{n}$ and $\psi_{n}: R_{n} \simeq S_{n}$, and the embeddings $U_{n} \rightarrow U_{n+1}, X_{n} \rightarrow X_{n+1}$, etc.

Let us denote by $\widetilde{R}$ and $\widetilde{U}$ the formal schemes obtained by completing $R \times \mathbb{A}^{1}$ and $U \times$ $\mathbb{A}^{1}$ respectively along the inverse images of $u_{0} \in U$, and by $\widetilde{S}$ and $\widetilde{X}$ the formal schemes obtained by completing $S \times \mathbb{A}^{1}$ and $X \times \mathbb{A}^{1}$ respectively along the inverse images of the origin in $X$. The structure maps of the schemes in groupoids $R_{n} \rightrightarrows U_{n}$ and $S_{n} \rightrightarrows X_{n}$ pass to the limit, yielding formal schemes in groupoids $\widetilde{R} \rightrightarrows \widetilde{U}$ and $\widetilde{S} \rightrightarrows \widetilde{X}$. The isomorphisms $\phi_{n}: U_{n} \simeq X_{n}$ and $\psi_{n}: R_{n} \simeq S_{n}$ give isomorphisms of formal schemes $\widetilde{\phi}: \widetilde{U} \simeq \widetilde{X}$ and $\widetilde{\psi}: \widetilde{R} \simeq \widetilde{S}$, yielding an isomorphism of formal schemes in groupoids of $\widetilde{R} \rightrightarrows \widetilde{U}$ with $\widetilde{S} \rightrightarrows \widetilde{X}$.

Denote by $I_{\widetilde{U}}$ and $I_{\widetilde{R}}$ the sheaves of ideals of the inverse images of $\mathcal{Y} \times\{0\} \subseteq \mathcal{X} \times \mathbb{A}^{1}$ in $\widetilde{U}$ and $\widetilde{R}$ respectively, and by $J_{\widetilde{U}}$ and $J_{\widetilde{R}}$ the pushforwards to $U$ and $\widetilde{R}$ of the sheaves of ideals of the pullbacks of the inverse images of $\mathcal{Y} \times\{0\}$ in the inverse images of $\mathcal{X} \times\{0\}$. Analogously, denote by $I_{\widetilde{X}}$ and $I_{\widetilde{S}}$ the sheaves of ideals of the inverse images of $[Y / G] \times\{0\} \subseteq[X / G] \times \mathbb{A}^{1}$ in $\widetilde{X}$ and $\widetilde{S}$ respectively, and by $J_{\widetilde{X}}$ and $J_{\widetilde{S}}$ the pushforwards to $\widetilde{X}$ and $\widetilde{S}$ of the sheaves of ideals of the pullbacks of the inverse images of $[Y / G] \times\{0\}$ in the inverse images of $[X / G] \times\{0\}$.

The natural morphisms $\tilde{u}: \widetilde{U} \rightarrow U \times \mathbb{A}^{1}, \tilde{r}: \widetilde{R} \rightarrow R \times \mathbb{A}^{1}, \tilde{x}: \tilde{X} \rightarrow X \times \mathbb{A}^{1}$ and $\tilde{s}: \widetilde{S} \rightarrow S \times \mathbb{A}^{1}$ are flat. Furthermore, the inverse images of $\mathcal{Y} \times\{0\} \subseteq \mathcal{X} \times \mathbb{A}^{1}$ and of $\mathcal{X} \times\{0\} \subseteq \mathcal{X} \times \mathbb{A}^{1}$ in $\widetilde{U}$ and $\widetilde{R}$ and the inverse images of $[Y / G] \times\{0\} \subseteq[X / G] \times \mathbb{A}^{1}$ 
and of $[X / G] \times\{0\} \subseteq[X / G] \times \mathbb{A}^{1}$ in $\widetilde{U}$ and $\widetilde{R}$ correspond under $\widetilde{\phi}$ and $\widetilde{\psi}$; hence for each $m \geq 0$ we obtain canonical isomorphisms of coherent sheaves

$$
\begin{array}{ll}
\tilde{u}^{*} I_{U}^{m} \simeq \tilde{\phi}^{*} \widetilde{x}^{*} I_{X}^{m}, & \widetilde{r}^{*} I_{R}^{m} \simeq \widetilde{\psi}^{*} \widetilde{S}^{*} I_{S}^{m}, \\
\tilde{u}^{*} J_{U}^{m} \simeq \widetilde{\phi}^{*} \widetilde{x}^{*} J_{X}^{m}, & \widetilde{r}^{*} J_{R}^{m} \simeq \widetilde{\psi}^{*} \widetilde{S}^{*} J_{S}^{m} .
\end{array}
$$

By restricting to $U_{n}$ and $R_{n}$ we obtain isomorphism of coherent sheaves

$$
\begin{aligned}
I_{U}^{m} \otimes_{\mathcal{O}_{U \times \mathbb{A}^{1}}} \mathcal{O}_{U_{n} \times \mathbb{A}^{1}} \simeq \phi_{n}^{*}\left(I_{X}^{m} \otimes_{\mathcal{O}_{X \times \mathbb{A}^{1}}} \mathcal{O}_{X_{n} \times \mathbb{A}^{1}}\right), \\
I_{R}^{m} \otimes_{\mathcal{O}_{R \times \mathbb{A}^{1}}} \mathcal{O}_{R_{n} \times \mathbb{A}^{1}} \simeq \phi_{n}^{*}\left(I_{S}^{m} \otimes_{\mathcal{O}_{X \times \mathbb{A}^{1}}} \mathcal{O}_{S_{n} \times \mathbb{A}^{1}}\right), \\
J_{U}^{m} \otimes_{\mathcal{O}_{U \times \mathbb{A}^{1}}} \mathcal{O}_{U_{n} \times \mathbb{A}^{1}} \simeq \phi_{n}^{*}\left(J_{X}^{m} \otimes_{\mathcal{O}_{X \times \mathbb{A}^{1}}} \mathcal{O}_{X_{n} \times \mathbb{A}^{1}}\right), \\
J_{R}^{m} \otimes_{\mathcal{O}_{R \times \mathbb{A}^{1}}} \mathcal{O}_{R_{n} \times \mathbb{A}^{1}} \simeq \phi_{n}^{*}\left(J_{S}^{m} \otimes_{\mathcal{O}_{X \times \mathbb{A}^{1}}} \mathcal{O}_{S_{n} \times \mathbb{A}^{1}}\right)
\end{aligned}
$$

By summing up over all $m$ we obtain an isomorphism of the corresponding Rees algebras, which yield the desired isomorphisms $U_{n}^{\prime} \simeq X_{n}^{\prime}$ and $R_{n}^{\prime} \simeq S_{n}^{\prime}$.

\subsection{Conclusion of the proof}

Now we are ready to finish the proof of Lemma 5.1.

Consider the complement $\mathcal{F}$ of $(\mathcal{M} \backslash \mathcal{N})^{0}$ in $\mathcal{M} \backslash \mathcal{N}$. Since $\mathcal{N}$ is a divisor on $\mathcal{M}$, the closure of $\mathcal{F}$ in $\mathcal{M}$ will not contain $\mathcal{N}$. Hence, a general $k$-rational point of $\mathcal{N}$ has a neighborhood $U$ such that the inertia stack $\mathcal{I}_{\mathcal{M}}$ is étale over $U \backslash \mathcal{N}$. Thus it is enough to show that $\mathcal{I}_{\mathcal{M}}$ is étale over $\mathcal{M}$ at a general point of $\mathcal{N}$. For this it suffices to show that the locus of points of the inverse image $\mathcal{I}_{\mathcal{N}}$ of $\mathcal{N}$ in $\mathcal{I}_{\mathcal{M}}$ at which $\mathcal{I}_{\mathcal{M}}$ is étale over $\mathcal{M}$ surjects onto $\mathcal{Y}$.

Denote by $N$ the normal bundle of $Y$ in $X$, and by $M$ the deformation to the normal bundle. If $n_{0}$ is a general closed point of $N$, then the pullback $I_{M}$ of the inertia stack of $[M / G]$ to $M$ is étale at $n_{0}$. Notice that $I_{M}$ is étale at a general closed point of the fiber of $N$ over any $y_{0} \in Y(k)$, since the action of $G$ on $Y$ is trivial. Hence, translation by any closed point of $Y$ is $G$-equivariant.

We know that $\mathcal{I}_{[M / G]}$ is étale over $\mathcal{M}$ at a general $k$-rational point of $\mathcal{I}_{[M / G]}$ lying over the image of the origin in $[N / G] \subseteq[M / G]$; let $v \in \mathcal{I}_{\mathcal{M}}(k)$ be a general $k$-rational point lying over $\mathcal{N}$, and let us show that $\mathcal{I}_{\mathcal{M}} \rightarrow \mathcal{M}$ is étale at $v$. For this we use the infinitesimal criterion for étaleness. Let $A$ be a finite $k$-algebra with residue field equal to $k$, let $I$ be a proper ideal in $A$, and consider a commutative diagram

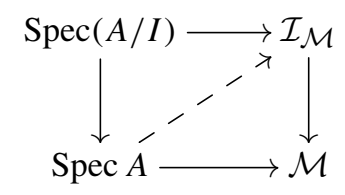

in which the composite $\operatorname{Spec} k \subseteq \operatorname{Spec} A \rightarrow \mathcal{I}_{\mathcal{M}}$ is isomorphic to $v$; we need to show that we can fill in the dashed arrow in a unique way. For $n \gg 0$, the morphism Spec $A \rightarrow \mathcal{M}$ 
factors through $\mathcal{M}_{n}$. Since $\mathcal{I}_{\mathcal{M}_{n}}=\mathcal{M}_{n} \times \mathcal{M}_{\mathcal{M}}$, the square above factors through a square

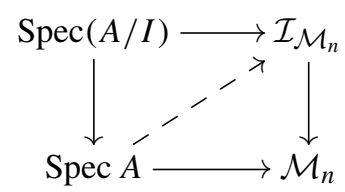

in which again we have to show the existence and uniqueness of the lifting. However, the isomorphism $\mathcal{M}_{n} \simeq\left[M_{n} / G\right]$ induces an isomorphism of the morphism $\mathcal{I}_{\mathcal{M}_{n}} \rightarrow \mathcal{M}_{n}$ with $\mathcal{I}_{\left[M_{n} / G\right]} \rightarrow\left[M_{n} / G\right]$; we know that $\mathcal{I}_{\left[M_{n} / G\right]}=\left[M_{n} / G\right] \times_{[M / G]} \mathcal{I}_{[M / G]}$ is étale at the point corresponding to $v$. Hence the lifting exists and is unique.

This completes the proof of Lemma 5.1 and thus of Theorem 1.2.

\subsection{A conjectural generalization}

On the basis of examples, the following generalization of Theorem 1.2 seems plausible.

Conjecture 5.4. Let $\mathcal{X}$ be an amenable stack over $k$. Let $L$ be an extension of $k$, and let $\xi$ be an object of $\mathcal{X}(\operatorname{Spec} L)$. Then

$$
\operatorname{ed}_{k} \xi \leq \operatorname{ged}_{k} \mathcal{X}+\operatorname{dim} \mathrm{R}_{\mathrm{u}}\left(\underline{\text { Aut }}_{L} \xi\right)
$$

Here $\mathrm{R}_{\mathrm{u}} G$ denotes the unipotent radical of $G$, as in Section 2.1. Unfortunately, the approach used in this section breaks down in the more general setting of the above conjecture: if the stabilizer is not reductive, the slice theorem does not apply.

\section{Essential dimension of $\mathrm{GL}_{n}$-quotients}

Suppose that $G$ is a special affine algebraic group over $k$ acting on a scheme $X$ locally of finite type over $k$. For each field $L / k$ we have an equivalence between $[X / G](L)$ and the quotient category for the action of the discrete group $G(L)$ on the set $X(L)$; hence the essential dimension of $[X / G]$ equals the essential dimension of the functor of orbits

$$
\operatorname{Orb}_{G, X}: \text { Field } / k \rightarrow \text { Set }
$$

from the category Field/ $k$ of extensions of $k$ to the category of sets, sending $L$ to the set of orbits $\operatorname{Orb}_{G, X}(L):=X(L) / G(L)$; see [BRV11, Example 2.6].

For the rest of this section we will assume that $X$ is an integral scheme, locally of finite type and smooth over $k$, and $\mathrm{GL}_{n}$ acts on $X$ with generically finite stabilizers. Then the quotient stack $\left[X / \mathrm{GL}_{n}\right]$ is amenable; however the Genericity Theorem 1.2 does not tell us that $\operatorname{ed}_{k}\left[X / \mathrm{GL}_{n}\right]=\operatorname{ged}_{k}\left[X / \mathrm{GL}_{n}\right]$ because we are not assuming that the stabilizer of every point of $X$ is reductive. Nevertheless, in some cases one can still establish this equality by estimating $\mathrm{ed}_{k} \xi$ from above and proving, in an ad-hoc fashion, that $\operatorname{ed}_{k}(\xi) \leq$ $\operatorname{ged}_{k}\left[X / \mathrm{GL}_{n}\right]$ for every $\xi \in\left[X / \mathrm{GL}_{n}\right](L)$ whose automorphism group is not reductive. 
The rest of this section will be devoted to such estimates. These estimates will ultimately allow us to deduce Theorem 1.1 from Proposition 3.4.

For each positive integer $\lambda$, denote by $J_{\lambda}$ the $\lambda \times \lambda$ Jordan block with eigenvalue 0 , that is, the $\lambda \times \lambda$ matrix of the linear transformation $k^{\lambda} \rightarrow k^{\lambda}$ defined by $e_{1} \mapsto 0$ and $e_{i} \mapsto e_{i-1}$ for $i=2, \ldots, \lambda$, where $e_{1}, \ldots, e_{\lambda}$ is the canonical basis of $k^{\lambda}$. Let $\underline{\lambda}$ be a partition of $n$; that is, $\underline{\lambda}=\left(\lambda_{1}, \ldots, \lambda_{r}\right)$ is a non-increasing sequence of positive integers with $\lambda_{1}+\cdots+\lambda_{r}=n$. We denote by $A_{\underline{\lambda}}$ the $n \times n$ nilpotent matrix which is written in block form as

$$
A_{\underline{\lambda}}:=\left(\begin{array}{cccc}
J_{\lambda_{1}} & 0 & \ldots & 0 \\
0 & J_{\lambda_{2}} & \ldots & 0 \\
\vdots & \vdots & \ddots & \vdots \\
0 & 0 & \ldots & J_{\lambda_{r}}
\end{array}\right)
$$

Every nilpotent $n \times n$ matrix is conjugate to a unique $A_{\underline{\lambda}}$. Consider the 1-parameter subgroup $\omega_{\underline{\lambda}}: \mathbb{G}_{\mathrm{a}} \rightarrow \mathrm{GL}_{n}$ defined by $\omega_{\underline{\lambda}}(t)=\exp \left(t A_{\underline{\lambda}}\right)$. We will usually assume that $\underline{\lambda} \neq\left(1^{n}\right)$; under this assumption $\omega_{\underline{\lambda}}$ is injective. Denote by $N_{\underline{\lambda}}$ the normalizer of the image of $\omega_{\underline{\lambda}}$; the group $N_{\underline{\lambda}}$ acts on the fixed point locus $X^{\omega_{\underline{\lambda}}}$.

Lemma 6.1. Let $L$ be a field extension of $k$, and $\xi$ be an object of $\left[X / \mathrm{GL}_{n}\right](L)$ whose automorphism group scheme $\underline{\mathrm{Aut}}_{L} \xi$ is not reductive. Then

$$
\operatorname{ed}_{k} \xi \leq \max _{\underline{\lambda}} \operatorname{ed}_{k}\left[X^{\omega_{\underline{\lambda}}} / N_{\underline{\lambda}}\right]
$$

where the maximum is taken over all partitions $\underline{\lambda}$ of $n$ different from $\left(1^{n}\right)$.

Proof. Suppose $\xi$ corresponds to the $\mathrm{GL}_{n}$-orbit of a point $p \in X(L)$. The automorphism group scheme $\underline{\mathrm{Aut}}_{L} \xi$ is isomorphic to the stabilizer $G_{p}$ of $p$ in $\mathrm{GL}_{n}$. Since we are assuming that this group is not reductive, $G_{p}$ will contain a copy of $\mathbb{G}_{\mathrm{a}}$, which is conjugate to the image of $\omega_{\underline{\lambda}}$ for some $\underline{\lambda} \neq\left(1^{n}\right)$. After changing $p$ to a suitable $\mathrm{GL}_{n}$-translate, we may assume that the image of $\omega_{\underline{\lambda}}$ is contained in $G_{p}$; hence $p \in X^{\omega_{\lambda}}$. The composite $X^{\omega_{\lambda}} \hookrightarrow X \rightarrow\left[X / \mathrm{GL}_{n}\right]$ factors through $\left[X^{\omega_{\lambda}} / N_{\underline{\lambda}}\right]$; hence $\xi$ is in the essential image of $\left[X^{\omega_{\underline{\lambda}}} / N_{\underline{\lambda}}\right](L)$ in $\left[X / \mathrm{GL}_{n}\right](L)$, and $\operatorname{ed}_{k} \xi \leq \operatorname{ed}_{k}\left[X^{\omega_{\underline{\lambda}}} / N_{\underline{\lambda}}\right]$.

Lemma 6.2. $\operatorname{ed}_{k}\left[X^{\omega_{\underline{\lambda}}} / N_{\underline{\lambda}}\right] \leq \operatorname{dim} X^{\omega_{\underline{\lambda}}}$ for any $\underline{\lambda} \neq\left(1^{n}\right)$.

Proof. By Lemma 2.1, $N_{\underline{\lambda}}$ is special. Hence,

$$
\operatorname{ed}_{k}\left[X^{\omega_{\underline{\lambda}}} / N_{\underline{\lambda}}\right]=\operatorname{ed}_{k} \operatorname{Orb}_{N_{\underline{\lambda}}, X^{\omega_{\underline{\lambda}}}} \leq \operatorname{dim} X^{\omega_{\underline{\lambda}}}
$$

as claimed.

We now further specialize $X$ to the affine space $A_{n, d}$ of forms of degree $d$ in the $n$ variables $\underline{x}=\left(x_{1}, \ldots, x_{n}\right)$ over $k$. The general linear group $\mathrm{GL}_{n}$ acts on $A_{n, d}$ in the usual way, via $(A f)(\underline{x}):=f\left(\underline{x} \cdot A^{-1}\right)$ for any $A \in \mathrm{GL}_{n}$. We are now ready for the main result of this section. 
Theorem 6.3. Let $L$ be a field extension of $k$, and $\xi$ be an object of $\left[A_{n, d} / \mathrm{GL}_{n}\right](L)$ whose automorphism group scheme $\underline{\mathrm{Aut}}_{L} \xi$ is not reductive. Assume that either $d \geq 4$ and $n \geq 2$ or $d=3$ and $n \geq 3$. Then $\operatorname{ed}_{k} \xi \leq\left(\begin{array}{c}n+d-1 \\ d\end{array}\right)-n^{2}$.

Proof. By Lemma 6.1 it suffices to show that

$$
\operatorname{ed}_{k}\left[X^{\omega_{\lambda}} / N_{\underline{\lambda}}\right] \leq\left(\begin{array}{c}
n+d-1 \\
d
\end{array}\right)-n^{2}
$$

for any $\underline{\lambda} \neq\left(1^{n}\right)$. The space $A_{n, d}^{\omega_{\lambda}}$ consists of the forms $f(\underline{x})$ such that

$$
f\left(x \exp \left(-t A_{\underline{\lambda}} x\right)\right)=f(\underline{x}) .
$$

By differentiating and applying the chain rule, this is equivalent to

$$
\nabla f(\underline{x}) \cdot A_{\underline{\lambda}}=0,
$$

where $\nabla f=\left(\partial f / \partial x_{1}, \ldots, \partial f / \partial x_{n}\right)$ is the gradient of $f$. We now proceed with the proof of (6.1) in three steps.

Case 1: $d \geq 4$. By Lemma 6.2 it suffices to show that

$$
\operatorname{dim} A_{n, d}^{\omega_{\lambda}} \leq\left(\begin{array}{c}
n+d-1 \\
d
\end{array}\right)-n^{2}
$$

for any $\underline{\lambda} \neq\left(1^{n}\right)$. For $\underline{\lambda} \neq\left(1^{n}\right)$ formula (6.2) tells us that $\partial f / \partial x_{1}$ is identically zero. In other words, $f(\underline{x})$ is a form in $x_{2}, \ldots, x_{n}$. Such forms lie in an affine subspace of $A_{n, d}$ isomorphic to $A_{n-1, d}$. Hence,

$$
\operatorname{dim} A_{n, d}^{\omega_{\lambda}} \leq \operatorname{dim} A_{n-1, d}=\left(\begin{array}{c}
n+d-2 \\
d
\end{array}\right),
$$

and it suffices to prove the inequality

$$
\left(\begin{array}{c}
n+d-1 \\
d
\end{array}\right)-\left(\begin{array}{c}
n+d-2 \\
d
\end{array}\right) \geq n^{2}
$$

or equivalently,

$$
\left(\begin{array}{c}
n+d-2 \\
d-1
\end{array}\right) \geq n^{2}
$$

Since $\left(\begin{array}{c}n+d-2 \\ d-1\end{array}\right)=\left(\begin{array}{c}n+d-2 \\ n-1\end{array}\right)$ is an increasing function of $d$ for any given $n \geq 1$, it suffices to prove (6.4) for $d=4$. In this case

$$
\left(\begin{array}{c}
n+d-2 \\
d-1
\end{array}\right)-n^{2}=\left(\begin{array}{c}
n+2 \\
3
\end{array}\right)-n^{2}=\frac{n(n-1)(n-2)}{6} \geq 0
$$

for any $n \geq 2$, as desired.

Case 2: $d=3$ and $\underline{\lambda} \neq\left(1^{n}\right)$ or $\left(2,1^{n-1}\right)$. Once again, it suffices to prove (6.3). If $\underline{\lambda} \neq\left(1^{n}\right)$ or $\left(2,1^{n-1}\right)$ then (6.2) shows that for every $f(\underline{x})$ in $A_{n, d}^{\omega_{\underline{\lambda}}}$ at least two of the 
partial derivatives, $\partial f / \partial x_{1}$ and $\partial f / \partial x_{i}$, are identically zero. For notational simplicity we will assume that $i=2$. Then $f(\underline{x})$ is a form in the variables $x_{3}, \ldots, x_{n}$. Hence,

$$
\operatorname{dim} A_{n, 3}^{\omega_{\lambda}} \leq \operatorname{dim} A_{n-2,3}=\left(\begin{array}{l}
n \\
3
\end{array}\right)=\left(\begin{array}{c}
n+2 \\
3
\end{array}\right)-n^{2}
$$

as desired.

Case 3: $d=3$ and $\underline{\lambda}=\left(2,1^{n-1}\right)$. Set $\omega:=\omega_{\left(2,1^{n-1}\right)}$ and $N:=N_{\left(2,1^{n-1}\right)}$. By Lemma 2.1(b), $N$ is a special group. Hence, we may identify the set of isomorphism classes in $\left[A_{n, 3}^{\omega} / N\right](K)$ with the set of $N(K)$-orbits in $A_{n, 3}^{\omega}(K)$, for every field $K / k$.

By (6.2), $A_{n, d}^{\omega}$ consists of degree $d$ forms $f\left(x_{1}, \ldots, x_{n}\right)$ such that $\partial f / \partial x_{1}=0$. That is, $f\left(x_{1}, \ldots, x_{n}\right) \in A_{n, d}^{\omega}$ if and only if $f$ is a form in the variables $x_{2}, \ldots, x_{n}$. Thus $A_{n, d}^{\omega}$ is an affine subspace of $A_{n, d}$ isomorphic to $A_{n-1, d}$. Our goal is to show that

$$
\operatorname{ed}_{k} f \leq\left(\begin{array}{c}
n+2 \\
3
\end{array}\right)-n^{2}
$$

for any $f(\underline{x}) \in A_{n, d}^{\omega}(K)$.

The normalizer $N$ contains a subgroup

$$
\Gamma \simeq \mathrm{GL}_{n-2} \ltimes \mathbb{G}_{\mathrm{a}}^{n-2}
$$

consisting of all matrices of the form

$$
\left(\begin{array}{ccccc}
1 & 0 & 0 & \ldots & 0 \\
0 & 1 & 0 & \ldots & 0 \\
0 & a_{3} & & & \\
\vdots & \vdots & & A & \\
0 & a_{n} & & &
\end{array}\right)
$$

where $\left(a_{3}, \ldots, a_{n}\right) \in \mathbb{G}_{\mathrm{a}}^{n-2}$ and $A \in \mathrm{GL}_{n-2}$. We may assume without loss of generality that the stabilizer of $f$ in $\Gamma$ does not contain a non-trivial unipotent subgroup. Indeed, if it does then $f$ is a $\Gamma$-translate of an element of $A_{n, 3}^{\omega_{\lambda}}$ for some $\underline{\lambda} \neq\left(1^{n}\right)$ or $\left(2,1^{n-1}\right)$. For such $f$ the inequality (6.5) was established in Case 2.

Since both $\Gamma$ and $N$ are special, it is obvious that the essential dimension of $f$, viewed as an element of $\left[A_{n, 3}^{\omega} / N\right](K)=\operatorname{Orb}_{N, A_{n, 3}^{\omega}}(K)$, is no greater than the essential dimension of $f$, viewed as an element of $\left[A_{n, 3}^{\omega} / \Gamma\right](K)=\operatorname{Orb}_{\Gamma, A_{n, 3}^{\omega}}(K)$. Since we are assuming that the stabilizer of $f$ in $\Gamma$ does not contain any non-trivial unipotent subgroups, the Genericity Theorem 1.2 tells us that

$$
\operatorname{ed}_{k} f \leq \operatorname{ged}_{k}\left[A_{n, 3}^{\omega} / \Gamma\right] .
$$

By Lemma 6.4 below for $n \geq 4$ the action of $\Gamma \subset \mathrm{GL}_{n-1}$ on the space $A_{n-1,3}$ of forms of degree 3 in the $n-1$ indeterminates $x_{2}, \ldots, x_{n}$ is generically free. Thus $\left[A_{n, 3} / \Gamma\right]$ is an amenable stack and is generically a scheme. Consequently,

$$
\operatorname{ged}_{k}\left[A_{n, 3}^{\omega} / \Gamma_{n-2}\right]=\operatorname{dim} A_{n-1,3}-\operatorname{dim} \Gamma_{n-2}=\left(\begin{array}{c}
n+1 \\
3
\end{array}\right)-(n-2)^{2}-(n-2) .
$$


A simple computation shows that

$$
\left(\begin{array}{c}
n+1 \\
3
\end{array}\right)-(n-2)^{2}-(n-2) \leq\left(\begin{array}{c}
n+2 \\
3
\end{array}\right)-n^{2}
$$

for any $n \geq 4$; indeed,

$$
\left(\begin{array}{c}
n+2 \\
3
\end{array}\right)-n^{2}-\left(\left(\begin{array}{c}
n+1 \\
3
\end{array}\right)-(n-2)^{2}-(n-2)\right)=\frac{(n-2)(n-3)}{2}-1 \geq 0 .
$$

\section{Lemma 6.4.}

(a) Assume that the base field $k$ is algebraically closed and $G$ is a connected linear algebraic $k$-group such that $N \cap Z(G) \neq\{1\}$ for every closed normal subgroup $\{1\} \neq N \triangleleft G$. Here $Z(G)$ denotes the center of $G$. (For example, $G$ could be almost simple or $\mathrm{GL}_{n}$.) Let $\mathrm{H}_{1}, \mathrm{H}_{2}$ be closed subgroups of $\mathrm{G}$ such that $\mathrm{H}_{1}$ is finite and $\mathrm{H}_{2}$ contains no non-trivial central elements of $G$. Then for $g \in G(k)$ in general position, $H_{1} \cap g H_{2} g^{-1}=\{1\}$.

(b) Assume $d \geq 3$ and $n \geq 1$. Let $\mathrm{GL}_{n-1}$ be the subgroup of $\mathrm{GL}_{n}$ acting on the variables $x_{2}, \ldots, x_{n}$. Then for $f \in A_{n, d}$ in general position, $\operatorname{Stab}_{\mathrm{GL}_{n-1}}(f)=\{1\}$.

Proof. (a) Assume the contrary. Consider the natural (translation) action of $H_{1}$ on the homogeneous space $G / H_{2}$. By our assumption this action is not generically free. Since $H_{1}$ is finite, we conclude that this action is not faithful, i.e., some $1 \neq h \in H_{1}$ acts trivially on $G / H_{2}$. Then $h$ lies in $N=\bigcap_{g \in G} g H_{2} g^{-1}$. Consequently, $N$ is a non-trivial normal subgroup of $G$. By our assumption $N$ (and hence $H_{2}$ ) contains a non-trivial central element of $G$, a contradiction.

(b) We may assume that $k$ is algebraically closed. By [Ric72, Theorem A], there exists a subgroup $S_{n, d} \subset \mathrm{GL}_{n}$ and a dense open subset $U \subset A_{n, d}$ such that $\operatorname{Stab}_{\mathrm{GL}_{n}}(f)$ is conjugate to $S$ for every $f \in U$. Moreover, for $d \geq 3$ (and any $n \geq 1$ ) $S_{n, d}$ is a finite group; see Example 3.2.

Write $f\left(x_{1}, \ldots, x_{n}\right)=\sum_{i=0}^{d} x_{1}^{d-i} f_{i}\left(x_{2}, \ldots, x_{n}\right)$, where $f_{i}$ is a form of degree $i$ in $x_{2}, \ldots, x_{n}$. Clearly $g \in \mathrm{GL}_{n-1}$ stabilizes $f$ if and only if it stabilizes $f_{1}, f_{2}, \ldots, f_{d}$. In other words,

$$
\operatorname{Stab}_{\mathrm{GL}_{n-1}}(f)=\bigcap_{i=1}^{d} \operatorname{Stab}_{\mathrm{GL}_{n-1}}\left(f_{i}\right) .
$$

Moreover, each $\operatorname{Stab}_{\mathrm{GL}_{n-1}}\left(f_{i}\right)$ is a conjugate of $S_{n-1, i}$ in $\mathrm{GL}_{n-1}$. Thus it suffices to show that for $g_{1}, \ldots, g_{d}$ in general position in $\mathrm{GL}_{n-1}$,

$$
g_{1} S_{n-1,1} g_{1}^{-1} \cap \cdots \cap g_{d} S_{n-1, d} g_{d}^{-1}=\{1\} .
$$

This is a consequence of part (a), with $G=\mathrm{GL}_{n}, H_{1}=S_{n-1,1}$ and $H_{2}=S_{n-1, d}$.

Remark 6.5. We note that if $d \geq 4$ and $n \geq 3$ or $d=3$ and $n \geq 5$ then (6.6) is immediate, since $S_{n-1, d}=\{1\}$; see Example 3.2. However, this argument does not cover the cases where $d=3$ and $n=3$ or 4 , which are needed for the proof of Theorem 6.3 above. 


\section{Proof of Theorem 1.1}

Theorem 1.1(a) is an immediate consequence of what we have done so far. Indeed, by Proposition 3.4(a),

$$
\operatorname{ged}_{k}\left[A_{n, d} / \mathrm{GL}_{n}\right]=\left(\begin{array}{c}
n+d-1 \\
d
\end{array}\right)-n^{2}+1+\operatorname{cd}\left(\mathrm{GL}_{n} / \mu_{d}\right) .
$$

Thus it suffices to show that for any field extension $K / k$ and any $K$-point $\zeta$ of $\left[A_{n, d} / \mathrm{GL}_{n}\right](K)$, we have

$$
\operatorname{ed}_{k} \zeta \leq \operatorname{ged}_{k}\left[A_{n, d} / \mathrm{GL}_{n}\right]
$$

If the automorphism group scheme $\underline{\operatorname{Aut}}_{K}(\zeta)$ is reductive, this is a direct consequence of Theorem 1.2, and if $\underline{\mathrm{Aut}}_{K}(\zeta)$ is not reductive, then Theorem 6.3 tells us that

$$
\operatorname{ed}_{k} \zeta \leq\left(\begin{array}{c}
n+d-1 \\
d
\end{array}\right)-n^{2}<\operatorname{ged}_{k}\left[A_{n, d} / \mathrm{GL}_{n}\right]
$$

This completes the proof of Theorem 1.1(a).

The rest of this section will be devoted to proving Theorem 1.1(b). The main complication here is that the stack $\left[\mathbb{P}\left(A_{n, d}\right) / \mathrm{GL}_{n}\right]$ is not amenable (see Example 3.2) and thus our Genericity Theorem 1.2 does not apply. We will get around this difficulty by relating $\left[\mathbb{P}\left(A_{n, d}\right) / \mathrm{GL}_{n}\right]$ to the amenable stack $\left[\mathbb{P}\left(A_{n, d}\right) / \mathrm{PGL}_{n}\right]$.

Proposition 7.1. Let $\mathcal{X}=\left[\mathbb{P}\left(A_{n, d}\right) / \mathrm{GL}_{n}\right]$ and $\overline{\mathcal{X}}:=\left[\mathbb{P}\left(A_{n, d}\right) / \mathrm{PGL}_{n}\right]$, with the natural projection $\phi: \mathcal{X} \rightarrow \overline{\mathcal{X}}$. Then for any extension $L / k$ and any $L$-point $\xi: \operatorname{Spec} L \rightarrow \mathcal{X}$,

$$
\operatorname{ed}_{k} \xi \leq \operatorname{ed}_{k} \phi(\xi)+\operatorname{cd}\left(\mathrm{GL}_{n} / \mu_{d}\right) .
$$

Proof. Note that $\mathcal{X}$ is a gerbe banded by $\mathbb{G}_{\mathrm{m}}$ over $\overline{\mathcal{X}}$.

By the definition of $\operatorname{ed}_{k} \phi(\xi)$ there exists an intermediate field $k \subset K \subset L$ such that $\phi(\xi)$ descends to Spec $K$ and $\operatorname{trdeg}_{k} K=\operatorname{ed}_{k} \phi(\xi)$. Moreover, $\xi: \operatorname{Spec} L \rightarrow \mathcal{X}_{K}$ factors through a point $\xi_{0}:$ Spec $L \rightarrow \mathcal{X}_{K}$, as in the diagram below.

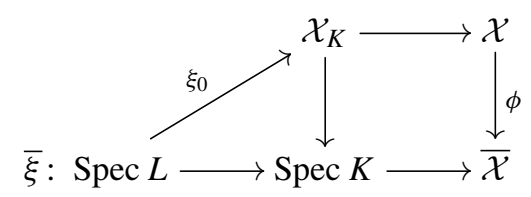

Note that $\mathcal{X}_{K}$ is a $\mathbb{G}_{\mathrm{m}}$-gerbe over $K$. So $\xi_{0}$ (and hence $\xi$ ) descends to some intermediate subfield of $K \subset K_{0} \subset L$ such that $\operatorname{trdeg}_{K} K_{0} \leq \operatorname{ed}_{k}\left(\mathcal{X}_{K}\right)=\operatorname{cd}\left(\mathcal{X}_{K}\right)$, where the last equality follows from Proposition 2.3(a). Let $\eta$ be the generic point of $\overline{\mathcal{X}}$. We know that the Brauer class of $\mathcal{X}_{\eta}$ has index dividing $n$ and exponent dividing $d$; see the proof of Proposition 3.4. By Lemma 2.4 the same is true of the Brauer class of $\mathcal{X}_{K}$. Therefore, by Lemma 2.2(c), $\operatorname{cd}\left(\mathcal{X}_{K}\right) \leq \operatorname{cd}\left(\mathrm{GL}_{n} / \mu_{d}\right)$. In summary,

$$
\begin{aligned}
\operatorname{ed}_{k} \xi & \leq \operatorname{trdeg}_{k} K_{0}=\operatorname{trdeg}_{k} K+\operatorname{trdeg}_{K} K_{0} \leq \operatorname{ed}_{k} \phi(\xi)+\operatorname{cd}\left(\mathcal{X}_{K}\right) \\
& \leq \operatorname{ed}_{k} \phi(\xi)+\operatorname{cd}\left(\mathrm{GL}_{n} / \mu_{d}\right),
\end{aligned}
$$

as claimed. 
Proof of Theorem 1.1(b). Let $\mathcal{X}:=\left[\mathbb{P}\left(A_{n, d}\right) / \mathrm{GL}_{n}\right], \overline{\mathcal{X}}:=\left[\mathbb{P}\left(A_{n, d}\right) / \mathrm{PGL}_{n}\right]$, and $\mathcal{Y}:=$ $\left[A_{n, d} \backslash\{0\} / \mathrm{GL}_{n}\right]$, and consider the following diagram of natural maps:

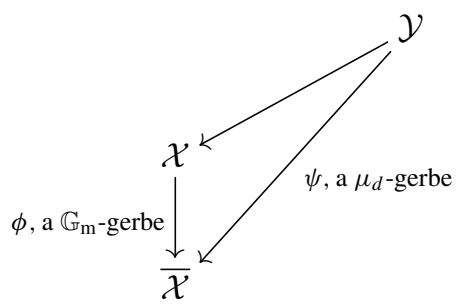

In view of Proposition 7.1, it suffices to show that for every field extension $L / k$ and every $L$-point $\xi:$ Spec $L \rightarrow \mathcal{X}$,

$$
\operatorname{ed}_{k} \phi(\xi) \leq\left(\begin{array}{c}
n+d-1 \\
d
\end{array}\right)-n^{2} .
$$

Recall from Example 3.2 that under our assumptions on $n$ and $d$, we know that $\overline{\mathcal{X}}=$ $\left[\mathbb{P}\left(A_{n, d}\right) / \mathrm{PGL}_{n}\right]$ is amenable and is generically a scheme of dimension

$$
\operatorname{dim} \mathbb{P}\left(A_{n, d}\right)-\operatorname{dim} \mathrm{PGL}_{n}=\left(\begin{array}{c}
n+d-1 \\
d
\end{array}\right)-n^{2} .
$$

If the automorphism group scheme $\underline{\mathrm{Aut}}_{L}(\phi(\zeta))$ is reductive then (7.1) holds by the Genericity Theorem 1.2 applied to $\overline{\mathcal{X}}$.

We may therefore assume that $\operatorname{Aut}_{L}(\phi(\zeta))$ is not reductive. Lift $\xi$ to some $\zeta$ : Spec $L$ $\rightarrow \mathcal{Y}$. (This can be done because $\mathcal{Y} \rightarrow \mathcal{X}$ is a $\mathbb{G}_{\mathrm{m}}$-torsor.) Since the automorphism group scheme $\operatorname{Aut}_{L}(\zeta)$ is contained in the preimage of $\operatorname{Aut}_{L}(\phi(\zeta))$ under the natural projection map $\mathrm{GL}_{n} \rightarrow \mathrm{PGL}_{n}$, we see that $\operatorname{Aut}_{L} \zeta$ is not reductive. Now ed $k \phi(\xi) \leq \operatorname{ed}_{k} \zeta$ and in view of Theorem 6.3

$$
\operatorname{ed}_{k} \zeta \leq\left(\begin{array}{c}
n+d-1 \\
d
\end{array}\right)-n^{2}
$$

This completes the proof of (7.1) and thus of Theorem 1.1(b).

\section{Small $n$ and $d$}

In this section we compute ed Forms $_{n, d}$ and ed Hypersurf $_{n, d}$ in the cases not covered by Theorem 1.1, building on the results of [BF03] and [BR05, Section 16].

To handle the case where $n=2$, we need the following variant of [BR05, Lemma 16.1]. The proof is similar; we reproduce it here, with the necessary modifications, for the sake of completeness.

Lemma 8.1. ed $_{k}$ Forms $_{2, d} \leq d-1$ and ed $_{k}$ Hypersurf $_{2, d} \leq d-2$ for any $d \geq 3$. 
Proof. Let $f\left(x_{1}, x_{2}\right)=a_{0} x_{1}^{d}+a_{1} x_{1}^{d-1} x_{2}+\cdots+a_{d} x_{2}^{d}$ be a non-zero binary form of degree $d$ over a field $K / k$. We claim that $f$ is equivalent (up to linear coordinate changes by elements of $\left.\mathrm{GL}_{2}(K)\right)$ to a binary form with (i) $a_{0}=0$ or $a_{1}=0$ and (ii) $a_{d-1}=0$ or $a_{d-1}=a_{d}$. In each case $f$ descends to the field $k\left(a_{0}, \ldots, a_{d}\right)$, and the hypersurface in $\mathbb{P}^{1}$ cut out by $f$ descends to the field $k\left(a_{i} / a_{j} \mid a_{j} \neq 0\right)$. If (i) and (ii) are satisfied then the transcendence degrees of these fields over $k$ are clearly $\leq d-1$ and $d-2$, respectively. So, the lemma follows from the claim.

To prove the claim, we first reduce $f$ to a form satisfying (i). If $a_{0}=0$, we are done. If $a_{0} \neq 0$, then performing the Tschirnhaus substitution

$$
x_{1} \mapsto x_{1}-\frac{a_{1}}{d a_{0}} x_{2}, \quad x_{2} \mapsto x_{2}
$$

we reduce $f$ to a binary form with $a_{1}=0$.

Now assume that $f$ satisfies (i). We want to further reduce it to a form satisfying both (i) and (ii). If $a_{d-1}=0$, we are done. If $a_{d-1} \neq 0$, rescale $x_{1}$ as follows:

$$
x_{1} \mapsto \frac{a_{d}}{a_{d-1}} x_{1}, \quad x_{2} \mapsto x_{2},
$$

to reduce $f$ to a form satisfying (i) and $a_{d-1}=a_{d}$. This completes the proof of the claim and the lemma.

Proposition 8.2. For any $n \geq 1$ and $d \geq 2$ we have

(a) ed $_{k}$ Forms $_{n, 1}=\operatorname{ed}_{k}$ Hypersurf $_{n, 1}=0$,

(b) ed $_{k}$ Forms $_{1, d}=1$ and ed $_{k}$ Hypersurf $_{1, d}=0$,

(c) ed $_{k}$ Forms $_{n, 2}=n$ and ed $_{k}$ Hypersurf $_{n, 2}=n-1$,

(d) ed $_{k}$ Forms $_{2,3}=2$ and ed $_{k}$ Hypersurf $_{2,3}=1$,

(e) ed $_{k}$ Forms $_{2,4}=3$ and ed $_{k}$ Hypersurf $_{2,4}=2$,

(f) ed $_{k}$ Forms $_{3,3}=4$ and ed $_{k}$ Hypersurf $_{3,3}=3$.

Proof. First we note that

$$
\text { ed }_{k} \text { Forms }_{n, d} \leq \text { ed }_{k} \text { Hypersurf }_{n, d}+1 .
$$

This is easy to see directly from the definition or, alternatively, as a special case of the Fiber Dimension Theorem [BRV11, Theorem 3.2(b)], applied to the representable morphism of quotient stacks $\left[A_{n, d} \backslash\{0\} / \mathrm{GL}_{n}\right] \rightarrow\left[\mathbb{P}\left(A_{n, d}\right) / \mathrm{GL}_{n}\right]$ of relative dimension 1 .

(a) Any non-zero linear form $F\left(x_{1}, \ldots, x_{n}\right)$ over any field $K / k$ is equivalent to $x_{1}$.

(b) Degree $d$ forms $f_{1}(x)=a x^{d}$ and $f_{2}(x)=b x^{d}$ over a field $K / k$ are equivalent if and only if $b=a c^{d}$ for some $c \in K^{*}=\mathrm{GL}_{1}(K)$. The assertions of part (b) follow easily from this.

(c) Any quadratic form $F\left(x_{1}, \ldots, x_{n}\right)$ over $K / k$ can be diagonalized, and hence is defined over an intermediate field $k \subset K_{0} \subset K$ such that $\operatorname{trdeg}_{k} K_{0} \leq n$. This implies that $\mathrm{ed}_{k}$ Forms $_{n, 2} \leq n$ and ed Hypersurf $_{n, 2} \leq n-1$. The opposite inequalities follow from [BR05, Proposition 16.2(b)]. 
(d) By Lemma 8.1, ed Forms $_{2,3} \leq 2$ and ed Hypersurf $_{2,3} \leq 1$, respectively. On the other hand, by [BR05, Proposition 16.2(c)], for the generic binary form $F_{\text {gen }}$ of degree 3 (as in (1.3)) and the hypersurface $H_{\text {gen }}$ it cuts out in $\mathbb{P}^{1}$, we have ed $F_{\text {gen }}=2$ and $\mathrm{ed}_{k} H_{\text {gen }}=1$.

Part (e) is proved in a similar manner, by combining Lemma 8.1 with [BR05, Proposition $16.2(\mathrm{~d})]$.

(f) The identity ed $_{k}$ Hypersurf $_{3,3}=3$ is the main result of [BF03]. By (8.1), $\mathrm{ed}_{k}$ Forms $_{3,3} \leq 4$.

In order to show that equality holds, it suffices to prove that the essential dimension $\operatorname{ged}_{k}\left[X_{3,3} / \mathrm{GL}_{3}\right]$ of the generic form $F_{\text {gen }}$ of degree 3 in three variables is at least 4 . For the purpose of proving this inequality we may replace $k$ by its algebraic closure $\bar{k}$ and thus assume without loss of generality that $k$ is algebraically closed. By [Ric72, Theorem A] the $\mathrm{GL}_{3}$-action on $X_{3,3}$ has a stabilizer in general position. Denote it by $S_{3,3}$, as in the proof of Lemma 6.4. As we mentioned there (and in Example 3.2), $S_{3,3}$ is a finite subgroup of $\mathrm{GL}_{3}$. Since the dimension of $\left[X_{3,3} / \mathrm{GL}_{3}\right]$ is 1 , by [BR05, Lemma 14.5(a) and Proposition 5.5(c)], ed $F_{\text {gen }} \geq \operatorname{ed}_{k} S_{3,3}+1$, where ed $S_{3,3}$ denotes the essential dimension of the finite group $S_{3,3}$ over $k$. (Note that in [BR05] the symbol $\phi_{3,3}$ was used in place of $F_{\text {gen }}$.) Thus it suffices to show that $\operatorname{ed}_{k} S_{3,3} \geq 3$.

To get a better idea about the structure of $S_{3,3}$, note that a $k$-point of $X_{3,3}$ in general position is represented by a scalar multiple of $x_{1}^{3}+x_{2}^{3}+x_{3}^{3}+3 a x_{1} x_{2} x_{3}$ for some $a \in k$. (Here we use the fact that $k$ is algebraically closed.) Hence, $S_{3,3}$ contains a nonabelian subgroup $H$ of order 27 , generated by diagonal matrices $\operatorname{diag}\left(\zeta_{1}, \zeta_{2}, \zeta_{3}\right)$, where $\zeta_{1}, \zeta_{2}$ and $\zeta_{3}$ are cube roots of unity satisfying $\zeta_{1} \zeta_{2} \zeta_{3}=1$, and the permutation matrices cyclically permuting $x_{1}, x_{2}$ and $x_{3}$. Now

$$
\operatorname{ed}_{k} S_{3,3} \geq \operatorname{ed}_{k} H \geq 3
$$

where the second inequality is a consequence of the Karpenko-Merkurjev theorem; see [MR10, Theorem 1.3]. This completes the proof of part (f).

Remark 8.3. (i) Since $S$ is a finite subgroup of $\mathrm{GL}_{3}$, it has a natural faithful 3-dimensional representation. Hence, $\operatorname{ed}_{k} S_{3,3} \leq 3$, and both inequalities in (8.2) are actually equalities.

(ii) The proof of part (e) shows that $\operatorname{ed}_{k} F_{\text {gen }}=4$, where $F_{\text {gen }}$ is the generic form of degree 3 in three variables, as in (1.3). This answers an open question posed after the statement of Proposition 16.2 in [BR05].

\section{Essential dimension of singular curves}

In this section we use our new Genericity Theorem 1.2 to strengthen [BRV11, Theorem 7.3] on the essential dimension of the stack on (not necessarily smooth) local complete intersection curves with finite automorphism group. Let us recall the set-up. Denote by $\mathfrak{M}_{g, n}$ the stack of all reduced $n$-pointed local complete intersection curves of genus $g$, that is, the algebraic stack over Spec $k$ whose objects over a $k$-scheme $T$ are finitely presented proper flat morphisms $\pi: C \rightarrow T$, together with $n$ sections $s_{1}, \ldots, s_{n}: T \rightarrow C$. 
Here $C$ is an algebraic space, the geometric fibers of $\pi$ are connected reduced local complete intersection curves of genus $g$, and the image of each $s_{i}$ is contained in the smooth locus of $C \rightarrow T$. (We do not require the images of the sections to be disjoint.)

The stack $\mathfrak{M}_{g, n}$ contains the stack $\mathcal{M}_{g, n}$ of smooth $n$-pointed curves of genus $g$ as an open substack (here the sections are supposed to be disjoint). By standard results in deformation theory, every reduced local complete intersection curve is unobstructed, and is a limit of smooth curves. Furthermore there is no obstruction to extending the sections, since these map into the smooth locus. Therefore $\mathfrak{M}_{g, n}$ is smooth and connected, and $\mathcal{M}_{g, n}$ is dense in $\mathfrak{M}_{g, n}$. However, the stack $\mathfrak{M}_{g, n}$ is very large (it is certainly not of finite type), and in fact it is very easy to see that its essential dimension is infinite. Assume that we are in the stable range, i.e., $2 g-2+n>0$ : then in [BRV11] we show that the essential dimension of the open substack $\mathfrak{M}_{g, n}^{\text {fin }}$ of $\mathfrak{M}_{g, n}$ of curves with finite automorphism group equals the essential dimension of $\mathcal{M}_{g, n}$.

Let $C$ be an object of $\mathfrak{M}_{g, n}$ defined over an algebraically closed field $K$. We say that $C$ is reductive if the automorphism group scheme $\underline{A u t}_{K} C$ is reductive. The marked curve $C$ is not reductive if and only if the smooth part $C_{\text {sm }} \subseteq C$ contains a component that is isomorphic to $\mathbb{A}_{K}^{1}$ and contains no marked points. A reductive object of $\mathfrak{M}_{g, n}$ is an object $C \rightarrow S$ whose geometric fibers over $S$ are reductive. It is not hard to see that the reductive objects form an open substack $\mathfrak{M}_{g, n}^{\text {red }}$ of $\mathfrak{M}_{g, n}$. Then our new genericity theorem applies, and allows one to conclude that the essential dimensions of $\mathfrak{M}_{g, n}^{\mathrm{red}}$ and of $\mathcal{M}_{g, n}$ are the same. From [BRV11, Theorem 1.2] we obtain the following.

Theorem 9.1. If $2 g-2+n>0$ and the characteristic of $k$ is 0 , then

$$
\operatorname{ed}_{k} \mathfrak{M}_{g, n}^{\mathrm{red}}= \begin{cases}2 & \text { if }(g, n)=(1,1) \\ 5 & \text { if }(g, n)=(2,0) \\ 3 g-3+n & \text { otherwise. }\end{cases}
$$

Acknowledgments. We are grateful to J. Alper and P. Brosnan for helpful discussions.

Research of Z. Reichstein was supported in part by NSERC Discovery and Accelerator Supplement grants.

Research of A. Vistoli was supported in part by the PRIN Project "Geometria sulle varietà algebriche", financed by MIUR.

\section{References}

[Alp09] Alper, J.: On the local quotient structure of Artin stacks. J. Pure Appl. Algebra 214, 1576-1591 (2010) Zbl 1205.14014 MR 2593684

[Art82] Artin, M.: Brauer-Severi varieties. In: Brauer Groups in Ring Theory and Algebraic Geometry (Wilrijk, 1981), Lecture Notes in Math. 917, Springer, Berlin, 194-210 (1982) Zbl 0536.14006 MR 0657430

[BF03] Berhuy, G., Favi, G.: Essential dimension: a functorial point of view (after A. Merkurjev). Documenta Math. 8, 279-330 (2003) Zbl 1101.14324 MR 2029168

[BF04] Berhuy, G., Favi, G.: Essential dimension of cubics. J. Algebra 278, 199-216 (2004) Zbl 1068.14035 MR 2068074 
[BR05] Berhuy, G., Reichstein, Z.: On the notion of canonical dimension for algebraic groups. Adv. Math. 198, 128-171 (2005) Zbl 1097.11018 MR 2183253

[BRV11] Brosnan, P., Reichstein, Z., Vistoli, A.: Essential dimension of moduli of curves and other algebraic stacks (with an appendix by N. Fakhruddin). J. Eur. Math. Soc. 13, 1079-1112 (2011) MR 2800485

[CGS58] Chevalley, C., Grothendieck, A., Serre, J.-P.: Séminaire C. Chevalley; 2e année: 1958. Anneaux de Chow et applications. Secrétariat mathématique, Paris (1958)

[CTKM07] Colliot-Thélène, J.-L., Karpenko, N. A., Merkur'ev, A. S.: Rational surfaces and the canonical dimension of the group PGL $_{6}$. Algebra i Analiz 19, 159-178 (2007) (in Russian) Zbl 1206.14070 MR 2381945

[Gab81] Gabber, O.: Some theorems on Azumaya algebras. In: The Brauer Group (Les Plans-sur-Bex, 1980), Lecture Notes in Math. 844, Springer, Berlin, 129-209 (1981) Zbl 0472.14013 MR 0611868

[Jan04] Jantzen, J. C.: Nilpotent orbits in representation theory. In: Lie Theory, Progr. Math. 228, Birkhäuser Boston, Boston, MA, 1-211 (2004) Zbl 1169.14319 MR 2042689

[KaMe06] Karpenko, N. A., Merkurjev, A. S.: Canonical p-dimension of algebraic groups. Adv. Math. 205, 410-433 (2006) Zbl 1119.14041 MR 2258262

[KeMo97] Keel, S., Mori, S.: Quotients by groupoids. Ann. of Math. (2) 145, 193-213 (1997) Zbl 0881.14018 MR 1432041

[LMB00a] Laumon, G., Moret-Bailly, L.: Champs algébriques. Ergeb. Math. Grenzgeb. 39, Springer, Berlin (2000) Zbl 0945.14005 MR 1771927

[MM64] Matsumura, H., Monsky, P.: On the automorphisms of hypersurfaces. J. Math. Kyoto Univ. 3, 347-361 (1963/1964) Zbl 0141.37401 MR 0168559

[McN06] McNinch, G.: On the centralizer of the sum of commuting nilpotent elements. J. Pure Appl. Algebra 206, 123-140 (2006) Zbl 1106.20036 MR 2220085

[MR10] Meyer, A., Reichstein, Z.: Some consequences of the Karpenko-Merkurjev theorem. Documenta Math. 2010, Extra Volume: Andrei A. Suslin's Sixtieth Birthday, 445-457 Zbl pre05827449 MR 2804261

[OS78] Orlik, P., Solomon, L.: Singularities. II. Automorphisms of forms. Math. Ann. 231, 229-240 (1977/78) Zbl 0352.14002 MR 0476735

[Ric72] Richardson, R. W., Jr.: Principal orbit types for algebraic transformation spaces in characteristic zero. Invent. Math. 16, 6-14 (1972) Z Zbl 0242.14010 MR 0294336

[San81] Sansuc, J.-J.: Groupe de Brauer et arithmétique des groupes algébriques linéaires sur un corps de nombres. J. Reine Angew. Math. 327, 12-80 (1981) Zbl 0468.14007 MR 0631309

[Ser03] Serre, J.-P.: Cohomological invariants, Witt invariants, and trace forms (notes by Skip Garibaldi). In: Cohomological Invariants in Galois Cohomology, Univ. Lecture Ser. 26, Amer. Math. Soc., Providence, RI, 1-100 (2003) MR 1999384

[TV13] Tossici, D., Vistoli, A.: On the essential dimension of infinitesimal group schemes. Amer. J. Math. 135, 103-114 (2013) Zbl pre06139349 MR 3022958 